\title{
De Novo Synthesis of Aceric Acid and an Aceric Acid Building Block
}

Mattie S. M. Timmer, Bridget L. Stocker, Peter H. Seeberger*

Laboratory for Organic Chemistry, Swiss Federal Institute of Technology (ETH) Zürich, Wolfgang-Pauli Strasse 10, 8093 Zurich, Switzerland

Experimental procedures for:

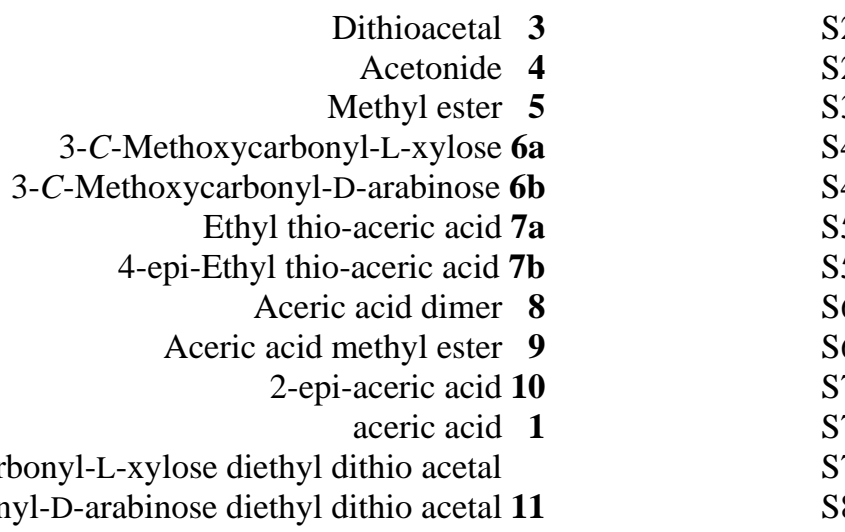

$\begin{array}{ll}\text { 5-deoxy-3-C-methoxycarbonyl-L-xylose diethyl dithio acetal } & \text { S7 } \\ \text { deoxy-3-C-methoxycarbonyl-D-arabinose diethyl dithio acetal 11 } & \text { S8 }\end{array}$

Spectra of compound: $\quad 3$

3

4

5

6a

$6 \mathbf{b}$

7a

$7 \mathbf{b}$

8

9

10

1

5-Deoxy-3-C-methoxycarbonyl-L-lyxose diethyl dithio acetal

11

Orthep Crystal structures:

$\begin{array}{rr}{ }^{1} \mathrm{H} & \mathrm{S} 9 \\ { }^{13} \mathrm{C} & \mathrm{S} 10 \\ { }^{1} \mathrm{H} & \mathrm{S} 11 \\ { }^{13} \mathrm{C} & \mathrm{S} 12 \\ { }^{1} \mathrm{H} & \mathrm{S} 13 \\ { }^{13} \mathrm{C} & \mathrm{S} 14 \\ { }^{1} \mathrm{H} & \mathrm{S} 15 \\ { }^{13} \mathrm{C} & \mathrm{S} 16 \\ { }^{1} \mathrm{H} & \mathrm{S} 17 \\ { }^{13} \mathrm{C} & \mathrm{S} 18 \\ { }^{1} \mathrm{H} & \mathrm{S} 19 \\ { }^{13} \mathrm{C} & \mathrm{S} 20 \\ { }^{1} \mathrm{H} & \mathrm{S} 21 \\ { }^{13} \mathrm{C} & \mathrm{S} 22 \\ { }^{1} \mathrm{H} & \mathrm{S} 23 \\ { }^{13} \mathrm{C} & \mathrm{S} 24 \\ { }^{13} \mathrm{H} & \mathrm{S} 25 \\ { }^{13} \mathrm{C} & \mathrm{S} 26 \\ { }_{3}^{1} \mathrm{H} & \mathrm{S} 27 \\ { }^{13} \mathrm{C} & \mathrm{S} 28 \\ { }^{1} \mathrm{H} & \mathrm{S} 29 \\ { }^{13} \mathrm{C} & \mathrm{S} 30 \\ { }^{1} \mathrm{H} & \mathrm{S} 31 \\ { }^{13} \mathrm{C} & \mathrm{S} 32 \\ { }^{1} \mathrm{H} & \mathrm{S} 33 \\ { }^{13} \mathrm{C} & \mathrm{S} 34 \\ { }^{13} \mathrm{C} & \mathrm{S} 35 \\ & \mathrm{~S} 36 \\ & \\ & \end{array}$


D-Arabinose diethyl dithio acetal (3). D-Arabinose (30 g, $150 \mathrm{mmol}$ ) was added to a vigorously stirred mixture of ethane thiol $(60 \mathrm{~mL})$ and conc. aq. $\mathrm{HCl}(60 \mathrm{~mL})$. As soon as the exothermic reaction started, the slightly pink solution was cooled $\left(0^{\circ} \mathrm{C}\right)$ and stirring was continued for 10 min upon which the product crystalized. The suspension was filtered and the residue was washed with cold water $(3 \times 50 \mathrm{~mL})$, dried by suction, washed with ether ( $3 \times 50 \mathrm{~mL})$, and dried again to obtain pure diethyl dithio acetal 3 (36.8 g, $144 \mathrm{mmol}, 72 \%$ ) as white platelets. Mp 125-126 ${ }^{\circ} \mathrm{C}$; $\alpha_{\mathrm{D}}{ }^{20}=-11$ (c 1.0, MeOH); IR $\left(\mathrm{CHCl}_{3}\right): 3560,3414,3051,2986,2929,1422,1265,1076,1024$, 940, $877 \mathrm{~cm}^{-1}$; ${ }^{1} \mathrm{H}-\mathrm{NMR}$ (300 MHz, CD $\mathrm{CDD}_{3}$ ): $\delta 4.03$ (d, $1 \mathrm{H}, J=9.2 \mathrm{~Hz}$ ), 3.97 (d, $1 \mathrm{H}, J=8.3$ Hz), 3.84 (d, $1 \mathrm{H}, J=9.2$ ), 3.78 (dd, $1 \mathrm{H}, J=2.9,10.7 \mathrm{~Hz}$ ), 3.68 (ddd, $1 \mathrm{H}, J=2.9,5.9,8.3 \mathrm{~Hz}$ ), 3.68 (dd, 1H, $J=5.9,10.8 \mathrm{~Hz}$ ), 2.78-2.58 (m, 4H), 1.24 (t, 3H, $J=7.4 \mathrm{~Hz}$ ), 1.23 (t, 3H, $J=7.4$ $\mathrm{Hz}) ;{ }^{13} \mathrm{C}-\mathrm{NMR}\left(75 \mathrm{MHz}, \mathrm{CD}_{3} \mathrm{OD}\right): \delta$ 73.1, 72.7, 71.9, 65.1, 56.2, 25.4, 15.0, 14.9; ESI-MS (m/z): $279.2[\mathrm{M}+\mathrm{Na}]^{+}, 535.2[2 \mathrm{M}+\mathrm{Na}]^{+}, 291.0[\mathrm{M}+\mathrm{Cl}]^{-}, 301.0\left[\mathrm{M}+\mathrm{HCO}_{2}\right]^{-}$; HRMS(EI) m/z calcld for $\mathrm{C}_{9} \mathrm{H}_{20} \mathrm{O}_{4} \mathrm{~S}_{2}^{+}: 256.0798$, obsd: 256.0797 .

2,3-O-Isopropylidine-D-arabinose diethyl dithio acetal (4). 2,2-Dimethoxypropane (40 mL, $325 \mathrm{~mL}, 2.25$ equiv.) and $p$-toluenesulfonic acid ( $0.5 \mathrm{~g}$, cat.) were added to a suspension of Darabinose diethyl dithioacetal 3 (36.8 g, $144 \mathrm{mmol})$ in acetone $(500 \mathrm{~mL})$. After obtaining a homogenous solution and stirring for an additional hour, the mixture was concentrated and the residue dissolved in a mixture of $\mathrm{MeOH}(500 \mathrm{~mL})$ and $\mathrm{H}_{2} \mathrm{O}(5 \mathrm{~mL})$. The solution was stirred for $2 \mathrm{~h}$ at $50^{\circ} \mathrm{C}$, sat. aq. $\mathrm{NaHCO}_{3}(100 \mathrm{~mL})$ was added and the mixture concentrated to $150 \mathrm{~mL}$. The residue was extracted with ethyl acetate $(3 \times 100 \mathrm{~mL})$, dried $\left(\mathrm{MgSO}_{4}\right)$ and concentrated. The resulting residue was triturated with dichloromethane and the solid D-arabinose diethyl dithioacetal (4.5 g; $17.6 \mathrm{mmol}$; 12\%) collected by filtration. Concentration of the mother liquor and purification of the residue by column chromatography (hexanes $\rightarrow$ ethyl acetate/hexanes, 1/3, v/v) gave homogeneous diol 4 (32 g, $108 \mathrm{mmol}, 75 \%)$ as a colorless oil. $\alpha_{D}{ }^{20}=59$ (c 1.0, $\left.\mathrm{CHCl}_{3}\right)$; IR $\left(\mathrm{CHCl}_{3}\right): 3606,3421,2991,2931,2873,1602,1455,1385,1374,1248,1161,1052$, 977, $881 \mathrm{~cm}^{-1}$; ${ }^{1} \mathrm{H}-\mathrm{NMR}\left(300 \mathrm{MHz}, \mathrm{CDCl}_{3}\right.$ ): $\delta 4.31$ (dd, $1 \mathrm{H}, J=3.8 \mathrm{~Hz}, J=6.9 \mathrm{~Hz}$ ), 4.08 (dd, $1 \mathrm{H}, J=6.9, J=7.0 \mathrm{~Hz}$ ), 4.01 (d, 1H, $J=3.8 \mathrm{~Hz}$ ), 3.81 (dd, $1 \mathrm{H}, J=2.8 \mathrm{~Hz}, J=10.5 \mathrm{~Hz}$ ), 3.73 (ddd, $J=2.8 \mathrm{~Hz}, J=5.4 \mathrm{~Hz}, J=7.0 \mathrm{~Hz}$ ), 3.67 (dd, $1 \mathrm{H}, J=5.4 \mathrm{~Hz}, J=10.5 \mathrm{~Hz}$ ), 2.99 (bs, 2H), 2.77-2.65 (m, 4H), 1.43 (s, 3H), 1.36 (s, 3H), 1.25 (t, 1H, $J=7.4 \mathrm{~Hz}$ ), 1.24 (t, 1H, $J=7.4 \mathrm{~Hz}$ );

${ }^{13} \mathrm{C}-\mathrm{NMR}\left(75 \mathrm{MHz}, \mathrm{CDCl}_{3}\right.$ ): $\delta$ 110.0, 83.2, 79.1, 73.0, 63.9, 53.1, 27.4, 27.1, 25.5, 25.2, 14.4; 
ESI-MS (m/z): $319.2[\mathrm{M}+\mathrm{Na}]^{+}, 615.2[2 \mathrm{M}+\mathrm{Na}]^{+}, 341.0\left[\mathrm{M}+\mathrm{HCO}_{2}\right]^{-}$; HRMS(EI) m/z calcld for $\mathrm{C}_{12} \mathrm{H}_{22} \mathrm{O}_{4} \mathrm{~S}_{2}^{+}$: 296.1111, obsd: 296.1109.

Methyl 2,3-O-isopropylidine-D-threuronate diethyl dithio acetal (5). A solution of $\mathrm{NaIO}_{4}$ (6.34 g, $30 \mathrm{mmol}, 1.4$ equiv.) in $\mathrm{H}_{2} \mathrm{O}(25 \mathrm{~mL})$ was added dropwise to a cooled $\left(0^{\circ} \mathrm{C}\right)$ and stirred solution of diol 4 (6.34 g, $21.4 \mathrm{mmol})$ in THF $(75 \mathrm{~mL})$. The ice bath was removed and the suspension stirred for 10 min before being quenched by the addition of conc. aq. sodium bicarbonate $(100 \mathrm{~mL})$ and extracted with ethyl acetate $(2 \times 100 \mathrm{~mL})$. The combined extracts were washed with brine $(200 \mathrm{~mL})$, dried $\left(\mathrm{MgSO}_{4}\right)$, filtered and concentrated. The resulting aldehyde was dissolved in dioxane $(100 \mathrm{~mL})$ and the solution was added to a stirred suspension of AgO, freshly made by the addition of a solution of $\mathrm{NaOH}$ ( $2.56 \mathrm{~g}, 64.2 \mathrm{mmol}, 3.0$ equiv.) in $\mathrm{H}_{2} \mathrm{O}$ (50 $\mathrm{mL}$ ) to a vigorously stirred solution of $\mathrm{AgNO}_{3}$ (7.28 g, $42.8 \mathrm{mmol}$, 2.0 equiv.) in $\mathrm{H}_{2} \mathrm{O}$ (50 mL). After $5 \mathrm{~min}$, the reaction mixture was filtered over celite and the filter washed with water. The aqueous solution was extracted with ether $(2 \times 100 \mathrm{~mL})$, acidified with $1 \mathrm{M}$ aq. $\mathrm{HCl}$ to $\mathrm{pH} 2$, and extracted with ethyl acetate $(3 \times 100 \mathrm{~mL})$. The combined organic extracts were dried $\left(\mathrm{MgSO}_{4}\right)$, filtered and concentrated. $\mathrm{KOH}$ (3.42 g, $56.1 \mathrm{mmol}, 2.8$ equiv.) was dissolved in a mixture of ether (15 mL), diethylene glycol monomethyl ether $(30 \mathrm{~mL})$ and $\mathrm{H}_{2} \mathrm{O}(15 \mathrm{~mL})$. This solution was placed in the distilling flask of a distillation setup equipped with a water bath at $70^{\circ} \mathrm{C}$, a Claisen adapter, a dropping funnel, water cooled condensor and a vacuum adapter for pressure relief, all without sharp edges or ground-glass joints. The acid was dissolved in ether $(90 \mathrm{~mL})$ and placed in the receiving flask. An ethereal solution of diazomethane ${ }^{1}$ was distilled by the dropwise addition of a solution of $N$-methyl- $N$-(p-tolylsulfonyl)nitrosamide (11.9 g, $55.6 \mathrm{mmol}, 2.6$ equiv.) in ether $(90 \mathrm{~mL})$. After complete addition of the ethereal solution, two additional portions of ether (10 $\mathrm{mL}$ ) were added slowly and the distillation continued until the distillate was colorless. After one hour, the reaction was quenched by the addition of acetic acid $(2 \times 1 \mathrm{~mL})$ in both the receiving and distillation flasks. After stirring for an additional 16h, the reaction mixture was concentrated and the residue was purified by column chromatography (hexanes $\rightarrow \mathrm{CH}_{2} \mathrm{Cl}_{2} /$ hexanes, $1 / 1, \mathrm{v} / \mathrm{v}$ ) to yield homogeneous methyl ester 5 (5.16 g, $18 \mathrm{mmol}$, 82\%) as a colorless oil. $\alpha_{\mathrm{D}}{ }^{20}=75$ (c 1.0, $\left.\mathrm{CHCl}_{3}\right)$; IR $\left(\mathrm{CHCl}_{3}\right): 2993,2931,1750,1439,1376,1263,1163,1109,876,816 \mathrm{~cm}^{-1}$; ${ }^{1} \mathrm{H}-\mathrm{NMR}$ (300 MHz, $\left.\mathrm{CDCl}_{3}\right): \delta 4.64$ (d, $\left.1 \mathrm{H}, J=6.8 \mathrm{~Hz}\right), 4.56(\mathrm{dd}, 1 \mathrm{H}, J=4.4 \mathrm{~Hz}, J=6.8 \mathrm{~Hz}), 3.98(\mathrm{~d}, 1 \mathrm{H}$,

\footnotetext{
${ }^{1}$ Caution: diazomethane is both explosive and carcinogenic and should always be treated with care. For the preparation and use of diazomethane see: De Boer, F.J.; Backer, H.J. Rec. Trav. Chim. Pays-Bas 1954, 73, 229-234.
} 
$J=4.4 \mathrm{~Hz}$ ), 3.79 (s, 3H), 2.77 (dq, 2H, $J=12.4 \mathrm{~Hz}, J=7.4 \mathrm{~Hz}$ ), 2.71 (dq, 2H, $J=12.3 \mathrm{~Hz}, J=$ $7.4 \mathrm{~Hz}$ ), 1.50 (s, 3H), 1.42 (s, 3H), 1.27 (t, 3H, $J=7.4 \mathrm{~Hz}$ ), 1.26 (t, 3H, $J=7.4 \mathrm{~Hz}$ ); ${ }^{13} \mathrm{C}-\mathrm{NMR}$ (75 MHz, $\left.\mathrm{CDCl}_{3}\right): \delta$ 170.9, 112.1, 82.2, 77.6, 52.8, 52.6, 52.6, 26.9, 25.9, 25.4, 25.0, 14.5, 14.4, 14.3; ESI-MS (m/z): $312.3\left[\mathrm{M}+\mathrm{NH}_{4}\right]^{+}, 317.3[\mathrm{M}+\mathrm{Na}]^{+}, 611.0[2 \mathrm{M}+\mathrm{Na}]^{+}$; HRMS(EI) m/z calcld for $\mathrm{C}_{12} \mathrm{H}_{22} \mathrm{O}_{4} \mathrm{~S}_{2}^{+}:$294.0955, obsd: 294.0962.

\section{5-Deoxy-3-C-methoxycarbonyl-2,3-O-isopropylidine-L-xylose diethyl dithio acetal (6a,} major) and 5-deoxy-3-C-methoxycarbonyl-2,3-O-isopropylidine-D-arabinose diethyl dithio acetal (6b, minor): BuLi (10.1 mL $1.6 \mathrm{M}$ in hexanes, $16.2 \mathrm{mmol}, 2.0$ equiv.) was added to a cooled $\left(-50^{\circ} \mathrm{C}\right)$, stirred solution of tetramethyl piperidine (2.87 mL, $17.0 \mathrm{mmol}, 2.1$ equiv.) in THF $(100 \mathrm{~mL})$. After $30 \mathrm{~min}$, the reaction mixture was cooled further $\left(-78^{\circ} \mathrm{C}\right)$ and a solution of methyl ester 5 ( $2.38 \mathrm{~g}, 8.1 \mathrm{mmol})$ in THF ( $20 \mathrm{~mL}$ ) was added dropwise. After another $30 \mathrm{~min}$, acetaldehyde (2.26 mL, $40.4 \mathrm{mmol}, 5.0$ equiv.) was added and the reaction mixture stirred for two hours, allowing the temperature to rise to $-20^{\circ} \mathrm{C}$. The reaction was quenched by the addition of sat. aq. $\mathrm{NH}_{4} \mathrm{Cl}(100 \mathrm{~mL})$ and extracted with ethyl acetate $(2 \times 100 \mathrm{~mL})$. The combined organic extracts were dried $\left(\mathrm{MgSO}_{4}\right)$, filtered and concentrated. Purification of the residue by column chromatography (hexanes $\rightarrow 5 \%$ ethyl acetate in hexanes) gave first $\mathbf{6 b}$ ( $0.50 \mathrm{~g}, 1.5 \mathrm{mmol}, 18 \%$ ) and then 6a (2.02 g, 6.0 mmol, 74 \%). 6a: $\alpha_{\mathrm{D}}{ }^{20}=70$ (c 1.0, $\left.\mathrm{CHCl}_{3}\right)$; IR $\left(\mathrm{CHCl}_{3}\right)$ : 3578, 2988, 2931, 2873, 1750, 1720, 1455, 1435, 1382, 1259, 1143, 1101, 1034, 978, 894, $840 \mathrm{~cm}^{-1} ;{ }^{1} \mathrm{H}-$ NMR (300 MHz, CDCl $): \delta 4.76$ (d, 1H, $J=3.0 \mathrm{~Hz}$ ), 4.13 (dq, 1H, $J=9.9 \mathrm{~Hz}, J=6.4 \mathrm{~Hz}$ ), 4.03 (d, $1 \mathrm{H}, J=3.0 \mathrm{~Hz}$ ), 3.74 (s, 1H), 2.74 (dq, $1 \mathrm{H}, J=12.4 \mathrm{~Hz}, J=7.5 \mathrm{~Hz}$ ), 2.69 (dq, $1 \mathrm{H}, J=7.5 \mathrm{~Hz}$, $J=12.4 \mathrm{~Hz}$ ), 2.69 (dq, 1H, $J=7.4 \mathrm{~Hz}, J=11.8 \mathrm{~Hz}), 2.60$ (dq, $1 \mathrm{H}, J=7.4 \mathrm{~Hz}, J=11.8 \mathrm{~Hz}), 2.01$ (d, $1 \mathrm{H}, J=9.9 \mathrm{~Hz}$ ), 1.61 (d, 3H, $J=0.5 \mathrm{~Hz}$ ), 1.43 (d, 3H, $J=0.5 \mathrm{~Hz}$ ), 1.26 (t, 3H, $J=7.4 \mathrm{~Hz}$ ), 1.21 (t, 3H, $J=7.5 \mathrm{~Hz}), 1.20$ (d, 3H, $J=6.4 \mathrm{~Hz}$ ); ${ }^{13} \mathrm{C}-\mathrm{NMR}\left(75 \mathrm{MHz}, \mathrm{CDCl}_{3}\right): \delta 171.7,110.7$, 87.9, 83.0, 68.1, 52.4, 50.9, 26.9, 26.7, 25.9, 25.0, 18.9, 14.4, 13.7; ESI-MS (m/z): 699.0 [2M+Na] $]^{+}$; HRMS(EI) $\mathrm{m} / \mathrm{z}$ calcld for $\mathrm{C}_{14} \mathrm{H}_{26} \mathrm{O}_{5} \mathrm{~S}_{2}{ }^{+}: 338.1217$, obsd: 338.1217. 6b: $\alpha_{\mathrm{D}}{ }^{20}=24(\mathrm{c}$ 1.0, $\left.\mathrm{CHCl}_{3}\right)$; IR $\left(\mathrm{CHCl}_{3}\right): 3566,2989,2931,2872,1750,1737,1456,1436,1377,1262,1144$, 1098, 1073, 970, 891, $835 \mathrm{~cm}^{-1}$; ${ }^{1} \mathrm{H}-\mathrm{NMR}\left(300 \mathrm{MHz}, \mathrm{CDCl}_{3}\right.$ ): $\delta 4.58$ (d, $1 \mathrm{H}, J=6.5 \mathrm{~Hz}$ ), 4.51 (qd, 1H, $J=6.3 \mathrm{~Hz}, J=7.5 \mathrm{~Hz}$ ), 4.43 (d, 1H, $J=6.5 \mathrm{~Hz}$ ), 3.78 (s, 3H), 2.82 (dq, 1H, $J=12.4 \mathrm{~Hz}$, $J=7.4 \mathrm{~Hz}$ ), 2.82 (d, 1H, $J=7.7 \mathrm{~Hz}$ ), 2.77 (q, 2H, $J=7.4 \mathrm{~Hz}$ ), 2.68 (dq, 1H, $J=12.4 \mathrm{~Hz}, J=7.4$ Hz), 1.59 (bq, 3H, ${ }^{4} J=0.6 \mathrm{~Hz}$ ), 1.48 (bq, 3H, $\left.{ }^{4} J=0.6 \mathrm{~Hz}\right), 1.294$ (t, 1H, $J=7.4 \mathrm{~Hz}$ ), 1.295 (t, 
$1 \mathrm{H}, J=7.4 \mathrm{~Hz}), 1.16$ (d, 3H, $J=6.3 \mathrm{~Hz}) ;{ }^{13} \mathrm{C}-\mathrm{NMR}\left(75 \mathrm{MHz}, \mathrm{CDCl}_{3}\right): \delta 172.3,110.1,87.7,84.7$, 66.5, 52.5, 49.2, 26.8, 25.6, 24.8, 24.5, 18.8, 14.2; ESI-MS (m/z): 699.0 [2M+Na]+; HRMS(EI) $\mathrm{m} / \mathrm{z}$ calcld for $\mathrm{C}_{14} \mathrm{H}_{26} \mathrm{O}_{5} \mathrm{~S}_{2}{ }^{+}: 338.1217$, obsd: 338.1217 .

1-Deoxy-1-ethylthio- $\alpha, \beta$-L-aceric acid methyl ester (7a). Acetonide 6a (100 mg, $0.30 \mathrm{mmol}$ ) was dissolved in a mixture of TFA $(2.5 \mathrm{~mL})$ and $\mathrm{H}_{2} \mathrm{O}(2.5 \mathrm{~mL})$. After stirring for $2 \mathrm{~h}$ at room temperature the solvents were evaporated in vacuo and the residue was taken up in pure TFA and stirred for an additional hour. Concentration of the reaction mixture and purification of the residue by column chromatography (ethyl acetate) to give thioglycoside 7 a (69 mg, $0.30 \mathrm{mmol}$, 99\%) as a colorless oil. $\alpha_{\mathrm{D}}{ }^{20}=4.7\left(c \mathrm{1} .0, \mathrm{CHCl}_{3}\right)$; IR $\left(\mathrm{CHCl}_{3}\right)$ : 3520, 3007, 2931, 1732, 1602, 1439, 1380, 1264, 1147, 1063, 1012, 970, 909, $891 \mathrm{~cm}^{-1}$. $\alpha$-anomer: ${ }^{1} \mathrm{H}$-NMR (300 MHz, $\mathrm{CDCl}_{3}$ ): $\delta 5.55$ (d, 1H, $J=5.0 \mathrm{~Hz}$ ), $4.61(\mathrm{q}, 1 \mathrm{H}, J=6.3 \mathrm{~Hz}$ ), $4.28(\mathrm{dd}, 1 \mathrm{H}, J=5.0 \mathrm{~Hz}, J=6.3$ Hz), 3.87 (s, 3H), 3.18 (bs, 1H), 2.96 (bd, $1 \mathrm{H}, J=6.3 \mathrm{~Hz}$ ), 2.77 (dq, 1H, $J=12.7 \mathrm{~Hz}, J=7.4 \mathrm{~Hz}$ ), $2.71(\mathrm{dq}, 1 \mathrm{H}, J=12.7 \mathrm{~Hz}, J=7.4 \mathrm{~Hz}$ ), 1.32 (t, $3 \mathrm{H}, J=7.4 \mathrm{~Hz}), 1.24$ (d, $3 \mathrm{H}, J=6.4 \mathrm{~Hz}$ ); ${ }^{13} \mathrm{C}-$ NMR (75 MHz, $\left.\mathrm{CDCl}_{3}\right): \delta 171.8,89.8,83.4,81.7,77.3,53.4,25.8,15.4,12.8 ; \beta$-anomer: ${ }^{1} \mathrm{H}-$ NMR (300 MHz, CDCl $): \delta 4.94$ (d, 1H, $J=5.3 \mathrm{~Hz}$ ), 4.42 (q, 1H, $J=6.4 \mathrm{~Hz}$ ), 4.12 (dd, $1 \mathrm{H}, J=$ $5.2 \mathrm{~Hz}, J=5.3 \mathrm{~Hz}$ ), 3.88 (s, 3H), 3.35 (bs, 1H), 2.90 (bd, 1H, $J=5.2 \mathrm{~Hz}$ ), 2.70 (q, 2H, $J=7.4$ $\mathrm{Hz}), 1.32(\mathrm{t}, 3 \mathrm{H}, J=7.4 \mathrm{~Hz}), 1.29(\mathrm{~d}, 3 \mathrm{H}, J=6.4 \mathrm{~Hz}) ;{ }^{13} \mathrm{C}-\mathrm{NMR}\left(75 \mathrm{MHz}, \mathrm{CDCl}_{3}\right): \delta 172.3$, 87.3, 84.3, 83.2, 79.5, 53.4, 25.3, 15.1, 14.9; ESI-MS (m/z): 281.0 [M+HCO ${ }_{2}{ }^{-} ; \mathrm{HRMS}(\mathrm{ESI}) \mathrm{m} / \mathrm{z}$ calcld for $\mathrm{C}_{9} \mathrm{H}_{16} \mathrm{O}_{5} \mathrm{SNa}^{+}:$259.0611, obsd: 259.0613 .

1-Deoxy-4-epi-1-ethylthio-L-aceric acid methyl ester (7b). Acetonide $6 \mathbf{b}$ (38 mg, $0.11 \mathrm{mmol}$ ) was dissolved in a mixture of TFA $(2.5 \mathrm{~mL})$ and $\mathrm{H}_{2} \mathrm{O}(2.5 \mathrm{~mL})$. After stirring for $2 \mathrm{~h}$ at room temperature the solvents were evaporated in vacuo and the residue was taken up in pure TFA and stirred for an additional hour. Concentration of the reaction mixture and purification of the residue by column chromatography (ethyl acetate) to obtain thioglycoside $7 \mathbf{b}$ ( $25 \mathrm{mg}, 0.11 \mathrm{mmol}$, 99\%) as a colorless oil. $\alpha_{D}{ }^{20}=151\left(c\right.$ 0.5, $\left.\mathrm{CHCl}_{3}\right)$; IR $\left(\mathrm{CHCl}_{3}\right): 3600,3514,3007,2958,2931$, 2874, 1732, 1602, 1440, 1381, 1280, 1160, 1112, 1087, 970, $870 \mathrm{~cm}^{-1}$; ${ }^{1} \mathrm{H}-\mathrm{NMR}$ (300 MHz, $\left.\mathrm{CDCl}_{3}\right): \delta 5.22(\mathrm{~d}, 1 \mathrm{H}, J=6.7 \mathrm{~Hz}), 4.26(\mathrm{~d}, 1 \mathrm{H}, J=6.7 \mathrm{~Hz}), 4.23(\mathrm{q}, 1 \mathrm{H}, J=6.5 \mathrm{~Hz}), 3.93(\mathrm{~s}$, 3H), 2.77 (qd, $1 \mathrm{H}, J=7.4 \mathrm{~Hz}, J=12.9 \mathrm{~Hz}$ ), 2.71 (qd, $1 \mathrm{H}, J=7.4 \mathrm{~Hz}, J=12.9 \mathrm{~Hz}$ ), 1.33 (t, 3H, $J$ $=7.4 \mathrm{~Hz}$ ), 1.18 (d, 3H, $J=6.5 \mathrm{~Hz}$ ); ${ }^{13} \mathrm{C}-\mathrm{NMR}\left(75 \mathrm{MHz}, \mathrm{CDCl}_{3}\right): \delta$ 171.9, 87.7, 84.9, 84.1, 77.4, 
77.2, 53.3, 25.8, 15.1, 14.2; ESI-MS (m/z): 281.0 [M+HCO ${ }_{2}$; ; HRMS(ESI) m/z calcld for $\mathrm{C}_{9} \mathrm{H}_{16} \mathrm{O}_{5} \mathrm{SNa}^{+}: 259.0611$, obsd: 259.0613 .

\section{Dimethyl $\quad(2 S, 3 R, 3 a S, 4 a S, 6 S, 7 R, 7 a S, 8 a S)-3,7-d i h y d r o x y-2,6-d i m e t h y l-h e x a h y d r o-1,4,5,8-$} tetraoxa-s-indacene-3,7-dicarboxylate (8). Bis(trifluoroacetoxy)iodobenzene (193 mg, 0.45 mmol, 1.5 equiv.) was added to a mixture of dithiane $6 \mathbf{6 a}(101 \mathrm{mg}, 0.30 \mathrm{mmol})$ in acetonitrile/ $\mathrm{H}_{2} \mathrm{O}$ ( $3 \mathrm{~mL}, 85 / 15, \mathrm{v} / \mathrm{v}$ ). After stirring for $30 \mathrm{~min}$, the reaction mixture was concentrated and the residue dissolved in TFA (5 mL). After stirring for $30 \mathrm{~min}$ at $50^{\circ} \mathrm{C}$ the solvent was evaporated in vacuo and the residue purified by column chromatography (hexanes $\rightarrow$ hexanes/ethyl acetate, 1:4) to give aceric acid dimer 8 (41 mg, $0.12 \mathrm{mmol}, 52 \%$ ) as a colorless oil. Crystallization from methanol/water gave colorless needles. $\alpha_{\mathrm{D}}{ }^{20}=136$ (c 0.2, $\left.\mathrm{CHCl}_{3}\right)$; IR $\left(\mathrm{CHCl}_{3}\right): 3600,3008,2926$, 2854, 1731, 1602, 1515, 1439, 1380, 1291, 1248, 1166, 1140, 1090, 1046, 994, 958, $928 \mathrm{~cm}^{-1}$; ${ }^{1} \mathrm{H}-\mathrm{NMR}\left(300 \mathrm{MHz}, \mathrm{CDCl}_{3}\right): \delta 5.08(\mathrm{~d}, 2 \mathrm{H}, J=3.8 \mathrm{~Hz}), 4.81(\mathrm{q}, 2 \mathrm{H}, J=6.4 \mathrm{~Hz}), 3.86(\mathrm{~s}, 6 \mathrm{H})$, 3.82 (d, $2 \mathrm{H}, J=3.8 \mathrm{~Hz}$ ), 1.27 (d, $6 \mathrm{H}, J=6.4 \mathrm{~Hz}$ ); ${ }^{13} \mathrm{C}-\mathrm{NMR}$ (75 MHz, CDCl $)$ ): $\delta 170.5,96.8$, 83.0, 81.5, 77.8, 53.3, 12.0; ESI-MS (m/z): $366.3\left[\mathrm{M}+\mathrm{NH}_{4}\right]^{+}, 371.0[\mathrm{M}+\mathrm{Na}]^{+}, 393.0\left[\mathrm{M}+\mathrm{HCO}_{2}\right]^{-}$; HRMS(ESI) $\mathrm{m} / \mathrm{z}$ calcld for $\mathrm{C}_{14} \mathrm{H}_{20} \mathrm{O}_{10} \mathrm{Na}^{+}:$371.0949, obsd: 371.0951 .

$\alpha, \beta$-L-Aceric acid methyl ester (9) Bis(trifluoroacetoxy)iodobenzene (66 mg) and sodium bicarbonate (34.7 mg) were added to a mixture of acetonide 6 a (32 mg, $0.11 \mathrm{mmol}$ ) in $\mathrm{H}_{2} \mathrm{O}$ (1 $\mathrm{mL}$ ) and acetonitrile (4 mL). After stirring for ten min, the reaction mixture was concentrated and the residue dissolved in a mixture of TFA $(5 \mathrm{~mL})$ and $\mathrm{H}_{2} \mathrm{O}(5 \mathrm{~mL})$. After stirring for $30 \mathrm{~min}$ at $50^{\circ} \mathrm{C}$ the solvents were evaporated in vacuo and the residue purified by column chromatography (hexanes $\rightarrow$ hexanes/ethyl acetate, 1:2) to give methyl ester 9 (16 mg, $0.083 \mathrm{mmol}, 88 \%$ ) as a colorless oil. $\alpha_{\mathrm{D}}{ }^{20}=54.6\left(c\right.$ 1.0, $\left.\mathrm{CHCl}_{3}\right)$; IR $\left(\mathrm{CHCl}_{3}\right): 3600,3528,3415,3031,2957,2921,2854$, 1726, 1439, 1383, 1264, 1156, 1063, 1013, 970, $908 \mathrm{~cm}^{-1}$; ${ }^{1} \mathrm{H}-\mathrm{NMR}$ (300 MHz, MeOD): $\alpha-$ anomer: $\delta 5.42$ (d, 1H, $J=4.2 \mathrm{~Hz}), 4.64$ (q, $1 \mathrm{H}, J=6.3 \mathrm{~Hz}), 4.05$ (d, $1 \mathrm{H}, J=4.2 \mathrm{~Hz}$ ), 3.77 (s, 3H), 1.16 (d, 3H, $J=6.3 \mathrm{~Hz}$ ); $\beta$-anomer: $\delta 5.02(\mathrm{~d}, 1 \mathrm{H}, J=2.1 \mathrm{~Hz}$ ), $4.52(\mathrm{q}, 1 \mathrm{H}, J=6.4 \mathrm{~Hz}$ ), 3.94 (d, $1 \mathrm{H}, J=2.1 \mathrm{~Hz}$ ), 3.76 (s, 3H), 1.24 (d, 3H, $J=6.4 \mathrm{~Hz}$ ); ${ }^{13} \mathrm{C}-\mathrm{NMR}(75 \mathrm{MHz}, \mathrm{MeOD}): \alpha-$ anomer: $\delta$ 172.2, 95.6, 82.4, 80.5, 76.9, 53.5, 13.6; $\beta$-anomer: $\delta$ 171.5, 102.8, 83.3, 81.8, 79.5, 53.6, 14.4; ESI-MS (m/z): $237.3\left[\mathrm{M}+\mathrm{HCO}_{2}\right]^{-}$; HRMS(ESI) m/z calcld for $\mathrm{C}_{7} \mathrm{H}_{12} \mathrm{O}_{6} \mathrm{Na}^{+}: 215.0526$, obsd: 215.0523. 
$\alpha, \beta$-L-2-Epi-Aceric acid (10) $\mathrm{NaOH}\left(1 \mathrm{~mL} 1 \mathrm{M}\right.$ in $\mathrm{H}_{2} \mathrm{O}, 1.0 \mathrm{mmol}, 16$ equiv.) was added to a solution of methyl ester 7 (12 mg, $62 \mu \mathrm{mol})$ in methanol $(4 \mathrm{~mL})$. After stirring for $30 \mathrm{~min}$ the reaction was neutralized by the addition of Amberlite IR 120, $\mathrm{H}^{+}$-form. Filtration and concentration gave 2-epi-aceric acid 10 (11 mg, $62 \mu \mathrm{mol}$, quant.) as a colorless oil. $\alpha_{\mathrm{D}}{ }^{20}=-3$ ( $c$ 1.0, MeOH); IR (neat): 3360, 2928, 2854, 1722, 1439, 1385, 1270, 1163, 1060, 1012, 895, 853 $\mathrm{cm}^{-1}$; $\alpha$-anomer: ${ }^{1} \mathrm{H}-\mathrm{NMR}$ (300 MHz, $\mathrm{D}_{2} \mathrm{O}$ ): $\delta 5.25$ (d, 1H, $J=5.0 \mathrm{~Hz}$ ), 4.69 (q, 1H, $J=6.4 \mathrm{~Hz}$ ), 4.54 (d, $1 \mathrm{H}, J=5.0 \mathrm{~Hz}$ ), 1.20 (d, 3H, $J=6.4 \mathrm{~Hz}$ ); ${ }^{13} \mathrm{C}-\mathrm{NMR}\left(75 \mathrm{MHz}, \mathrm{D}_{2} \mathrm{O}\right): \delta$ 174.8, 101.3, 82.7, 82.2, 79.2, 79.1, 13.2. $\beta$-anomer: ${ }^{1} \mathrm{H}-\mathrm{NMR}\left(300 \mathrm{MHz}, \mathrm{D}_{2} \mathrm{O}\right): \delta 5.33$ (d, $1 \mathrm{H}, J=5.4 \mathrm{~Hz}$ ), 4.64 (d, $1 \mathrm{H}, J=5.4 \mathrm{~Hz}), 4.39$ (q, 1H, $J=6.4 \mathrm{~Hz}), 1.26$ (d, 3H, $J=6.4 \mathrm{~Hz}) ;{ }^{13} \mathrm{C}-\mathrm{NMR}(75 \mathrm{MHz}$, $\left.\mathrm{D}_{2} \mathrm{O}\right): \delta$ 176.9, 95.5, 81.2, 75.9, 14.6; ESI-MS (m/z): $195.0[\mathrm{M}+\mathrm{OH}]^{-}, 223.0\left[\mathrm{M}+\mathrm{HCO}_{2}\right]^{-}$; HRMS(ESI) m/z calcld for $\mathrm{C}_{6} \mathrm{H}_{9} \mathrm{O}_{6}{ }^{-}: 177.0405$, obsd: 177.0408 .

$\boldsymbol{\alpha}, \boldsymbol{\beta}$-L-Aceric acid (1) $\mathrm{LiOH}\left(0.125 \mathrm{~mL} \mathrm{1M}\right.$ in $\mathrm{H}_{2} \mathrm{O}, 125 \mu \mathrm{mol}, 2$ equiv.) was added to a solution of methyl ester 9 (12 mg, $62 \mu \mathrm{mol})$ in $\mathrm{H}_{2} \mathrm{O}(1 \mathrm{~mL})$ and methanol $(1 \mathrm{~mL})$ at $0^{\circ} \mathrm{C}$. After stirring for 90 min, allowing the mixture to warm up to room temperature, the reaction was neutralized by the addition of Amberlite IR 120, $\mathrm{H}^{+}$-form. Filtration and concentration gave aceric acid 1 (11 mg, $62 \mu \mathrm{mol}$, quant.) as a colorless oil. $\alpha_{\mathrm{D}}{ }^{20}=-35.6$ (c 1.0, $\mathrm{CH}_{3} \mathrm{OH}$ ); IR (neat): 3375, 2927, 2854, 1722, 1439, 1385, 1270, 1163, 1060, 1012, 895, $853 \mathrm{~cm}^{-1} ;{ }^{1} \mathrm{H}-\mathrm{NMR}\left(300 \mathrm{MHz}, \mathrm{D}_{2} \mathrm{O}\right): \delta 5.49$ (d, $1 \mathrm{H}, J=4.4 \mathrm{~Hz}$ ), 5.19 (d, 1H, $J=2.8 \mathrm{~Hz}), 4.69$ (q, 1H, $J=6.4 \mathrm{~Hz}), 4.56$ (q, 1H, $J=6.5 \mathrm{~Hz}), 4.16$ (d, 1H, $J=4.4 \mathrm{~Hz}$ ), 4.05 (d, 1H, $J=2.8 \mathrm{~Hz}$ ), 1.26 (d, 1H, $J=6.5 \mathrm{~Hz}$ ), 1.19 (d, 1H, $J=6.4 \mathrm{~Hz}$ ); ); ${ }^{13} \mathrm{C}-\mathrm{NMR}\left(75 \mathrm{MHz}, \mathrm{D}_{2} \mathrm{O}\right): \delta$ 176.6, 176.6, 104.0, 98.4, 86.2, 85.4, 85.0, 80.9, 80.9, 79.6, 16.6, 15.3; ESI-MS (m/z): 177.0 [M-H]', 195.0 [M+OH]', 223.0 [M+HCO $]^{-} ; \mathrm{HRMS}(\mathrm{ESI}) \mathrm{m} / \mathrm{z}$ calcld for $\mathrm{C}_{6} \mathrm{H}_{9} \mathrm{O}_{6}^{-}: 177.0405$, obsd: 177.0407 .

5-deoxy-3-C-methoxycarbonyl-L-xylose diethyl dithio acetal. Acetonide 6a (80 mg, 0.24 mmol) was dissolved in a mixture of TFA $(2.5 \mathrm{~mL})$ and $\mathrm{H}_{2} \mathrm{O}(2.5 \mathrm{~mL})$. After stirring for $2 \mathrm{~h}$ at room temperature the solvents were evaporated in vacuo and the residue purified by column chromatography $\left(\mathrm{CH}_{2} \mathrm{Cl}_{2} \rightarrow 0.5 \% \mathrm{MeOH}\right.$ in $\left.\mathrm{CH}_{2} \mathrm{Cl}_{2}\right)$ to obtain the title compound (71 $\mathrm{mg}, 0.24$ mmol, quant.) as a colorless oil. $\alpha_{\mathrm{D}}{ }^{20}=-57$ (c 1.0, $\left.\mathrm{CHCl}_{3}\right)$; IR $\left(\mathrm{CHCl}_{3}\right)$ : 3512, 3032, 2929, 2854, 1728, 1602, 1458, 1437, 1378, 1266, 1156, 1022, 1005, 890, $821 \mathrm{~cm}^{-1}$; ${ }^{1} \mathrm{H}-\mathrm{NMR}$ (300 MHz, 
$\left.\mathrm{CDCl}_{3}\right): \delta 4.25$ (q, 1H, $\left.J=6.4 \mathrm{~Hz}\right), 4.12(\mathrm{~d}, 1 \mathrm{H}, J=7.8 \mathrm{~Hz}), 4.03$ (d, $\left.1 \mathrm{H}, J=7.8 \mathrm{~Hz}\right), 3.76(\mathrm{~s}$, 1H), 2.71 (dq, 1H, $J=12.4 \mathrm{~Hz}, J=7.5 \mathrm{~Hz}$ ), 2.70 (dq, $1 \mathrm{H}, J=7.5 \mathrm{~Hz}, J=12.4 \mathrm{~Hz}$ ), 2.65 (dq, 1H, $J=7.5 \mathrm{~Hz}, J=12.4 \mathrm{~Hz}$ ), $2.62(\mathrm{dq}, 1 \mathrm{H}, J=7.5 \mathrm{~Hz}, J=11.8 \mathrm{~Hz}), 1.26$ (t, 3H, $J=7.5 \mathrm{~Hz}), 1.24(\mathrm{t}$,

$3 \mathrm{H}, J=7.5 \mathrm{~Hz}$ ), 1.15 (d, 3H, $J=6.4 \mathrm{~Hz}$ ); ${ }^{13} \mathrm{C}-\mathrm{NMR}\left(75 \mathrm{MHz}, \mathrm{CDCl}_{3}\right): \delta$ 172.4, 79.7, 76.0, 71.9, 54.5, 52.6, 26.2, 24.3, 17.1, 14.5, 14.0; ESI-MS (m/z): $321.0[\mathrm{M}+\mathrm{Na}]^{+}, 619.0[2 \mathrm{M}+\mathrm{Na}]^{+}$; HRMS(ESI) $\mathrm{m} / \mathrm{z}$ calcld for $\mathrm{C}_{11} \mathrm{H}_{22} \mathrm{O}_{5} \mathrm{~S}_{2} \mathrm{Na}^{+}$: 321.0801, obsd: 321.0801 .

5-Deoxy-3-C-methoxycarbonyl-D-arabinose diethyl dithio acetal (11). Acetonide $6 \mathbf{b}$ (38 mg, $0.11 \mathrm{mmol})$ was treated with a mixture of TFA $(2.5 \mathrm{~mL})$ and $\mathrm{H}_{2} \mathrm{O}(2.5 \mathrm{~mL})$. After stirring for $2 \mathrm{~h}$ the solvents were evaporated in vacuo and the residue purified by column chromatography $\left(\mathrm{CH}_{2} \mathrm{Cl}_{2} \rightarrow 0.5 \% \mathrm{MeOH}\right.$ in $\left.\mathrm{CH}_{2} \mathrm{Cl}_{2}\right)$ to obtain 11 (35 mg, $0.11 \mathrm{mmol}$, quant.) as a colorless solid. Crystallization from ethyl acetate/hexanes gave white needles. $\alpha_{\mathrm{D}}{ }^{20}=-19$ (c 1.0, $\mathrm{CHCl}_{3}$ ); IR ( $\left.\mathrm{CHCl}_{3}\right)$ : 3504, 2989, 2976, 2931, 2873, 1729, 1602, 1458, 1438, 1379, 1248, 1147, 1087, 890 $\mathrm{cm}^{-1}$; ${ }^{1} \mathrm{H}-\mathrm{NMR}$ (300 MHz, CDCl $): \delta 4.24$ (dd, $1 \mathrm{H}, J=3.8 \mathrm{~Hz}, J=7.8 \mathrm{~Hz}$ ), 4.05 (bdq, $1 \mathrm{H}, J=6.5$ Hz, $J=8.4 \mathrm{~Hz}$ ), 4.02 (d, 1H, $J=7.8 \mathrm{~Hz}$ ), 3.82 (s, 1H), 3.68 (s, 1H), 3.47 (d, 1H, $J=3.8 \mathrm{~Hz}$ ), 2.72 (dq, $1 \mathrm{H}, J=7.5 \mathrm{~Hz}, J=11.9 \mathrm{~Hz}$ ), $2.71(\mathrm{dq}, 1 \mathrm{H}, J=7.5 \mathrm{~Hz}, J=12.4 \mathrm{~Hz}$ ), 2.68 (bd, $1 \mathrm{H}, J=8.4$ Hz), 2.66 (dq, 1H, $J=7.5 \mathrm{~Hz}, J=11.9 \mathrm{~Hz}), 2.61$ (dq, $1 \mathrm{H}, J=7.5 \mathrm{~Hz}, J=12.4 \mathrm{~Hz}), 1.26$ (t, 3H, $J$ $=7.5 \mathrm{~Hz}$ ), 1.24 (t, 3H, $J=7.5 \mathrm{~Hz}), 1.22$ (d, 3H, $J=6.5 \mathrm{~Hz}) ;{ }^{13} \mathrm{C}-\mathrm{NMR}\left(75 \mathrm{MHz}, \mathrm{CDCl}_{3}\right): \delta$ 173.5, 80.5, 72.7, 71.2, 54.4, 52.8, 25.9, 24.2, 17.5, 14.4, 14.0; ESI-MS (m/z): $321.0[\mathrm{M}+\mathrm{Na}]^{+}$, $619.0[2 \mathrm{M}+\mathrm{Na}]^{+}$; HRMS(ESI) m/z calcld for $\mathrm{C}_{11} \mathrm{H}_{22} \mathrm{O}_{5} \mathrm{~S}_{2} \mathrm{Na}^{+}$: 321.0801, obsd: 321.0808 . 

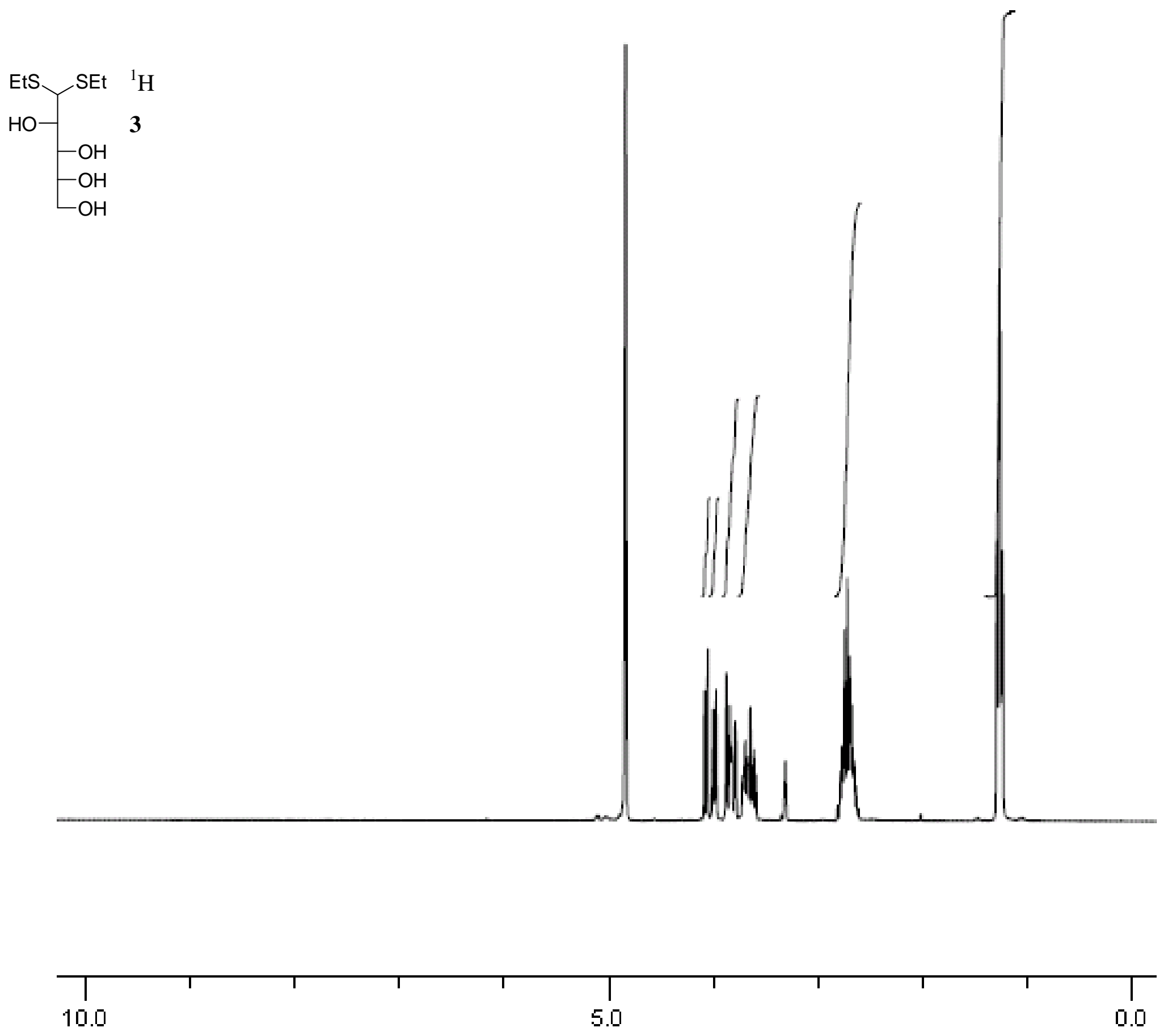


${ }^{\mathrm{EtS}}{ }^{\mathrm{SEt}}{ }^{13} \mathrm{C}$

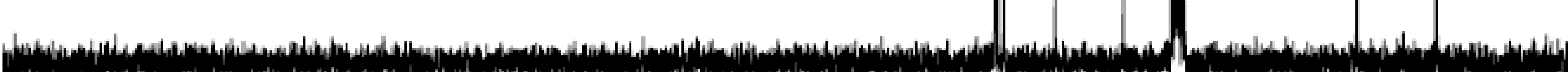

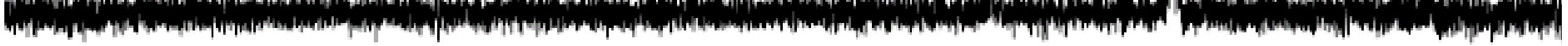

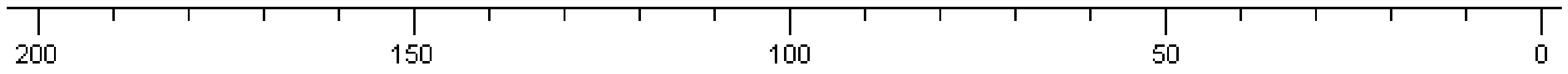



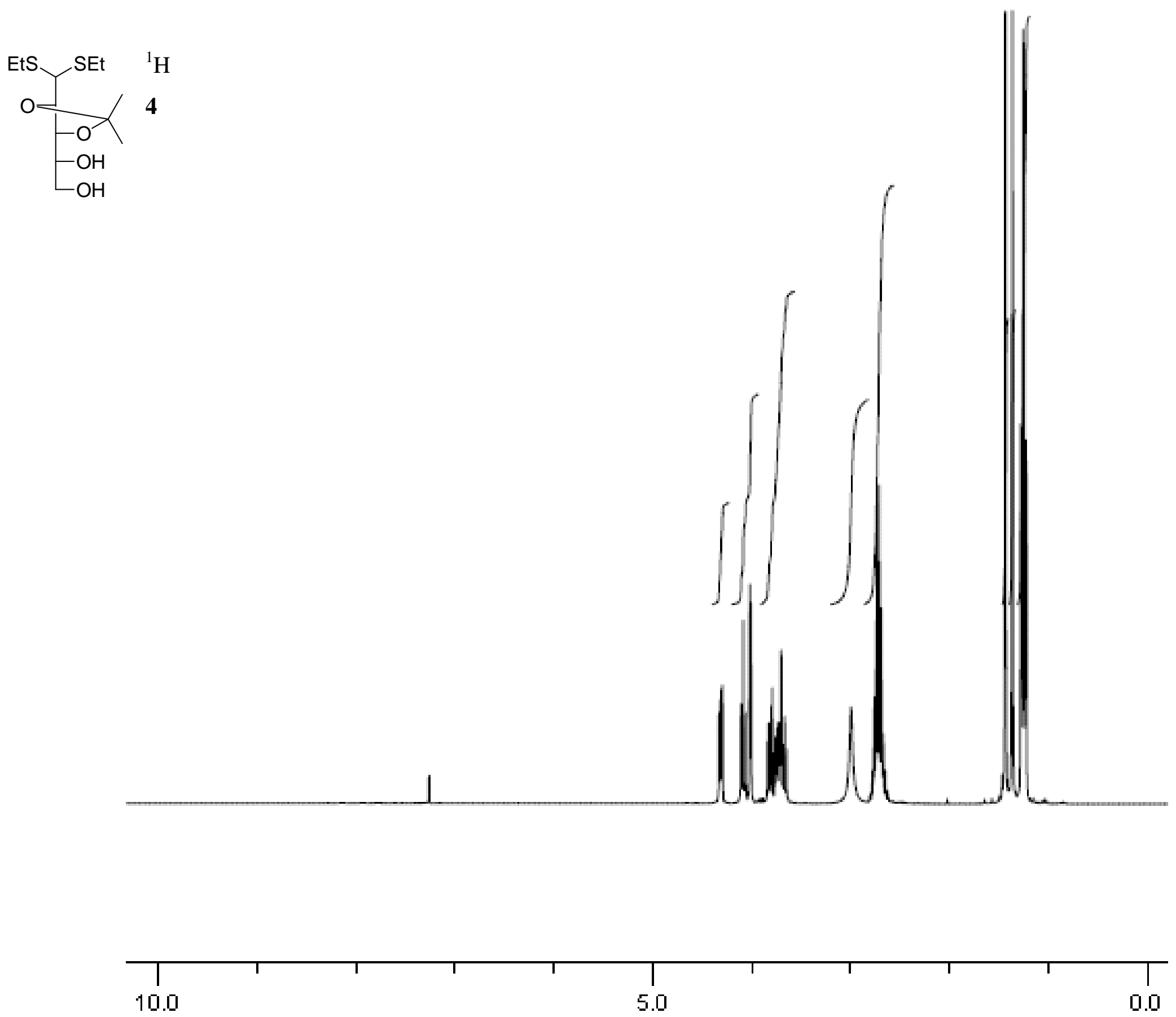

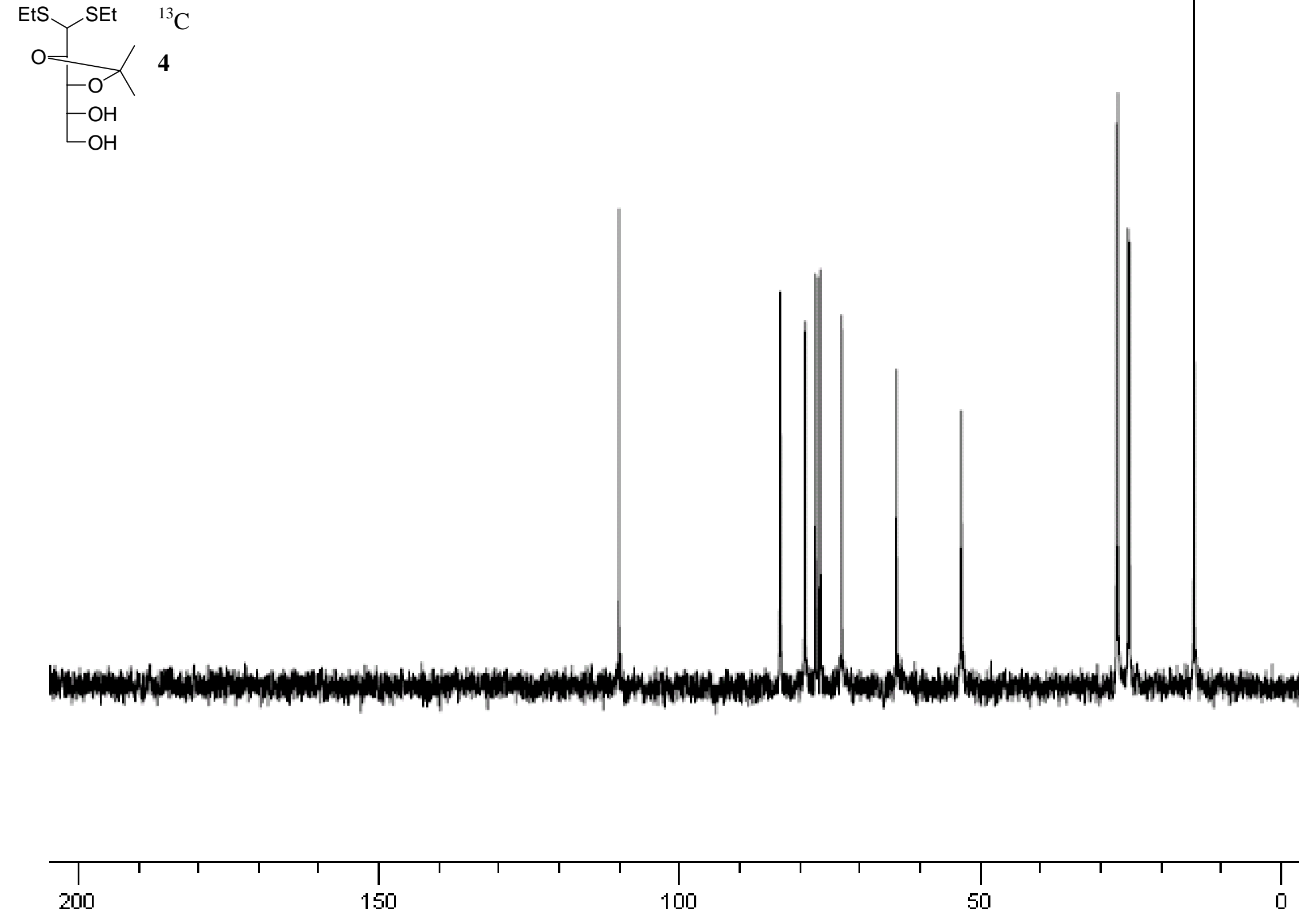

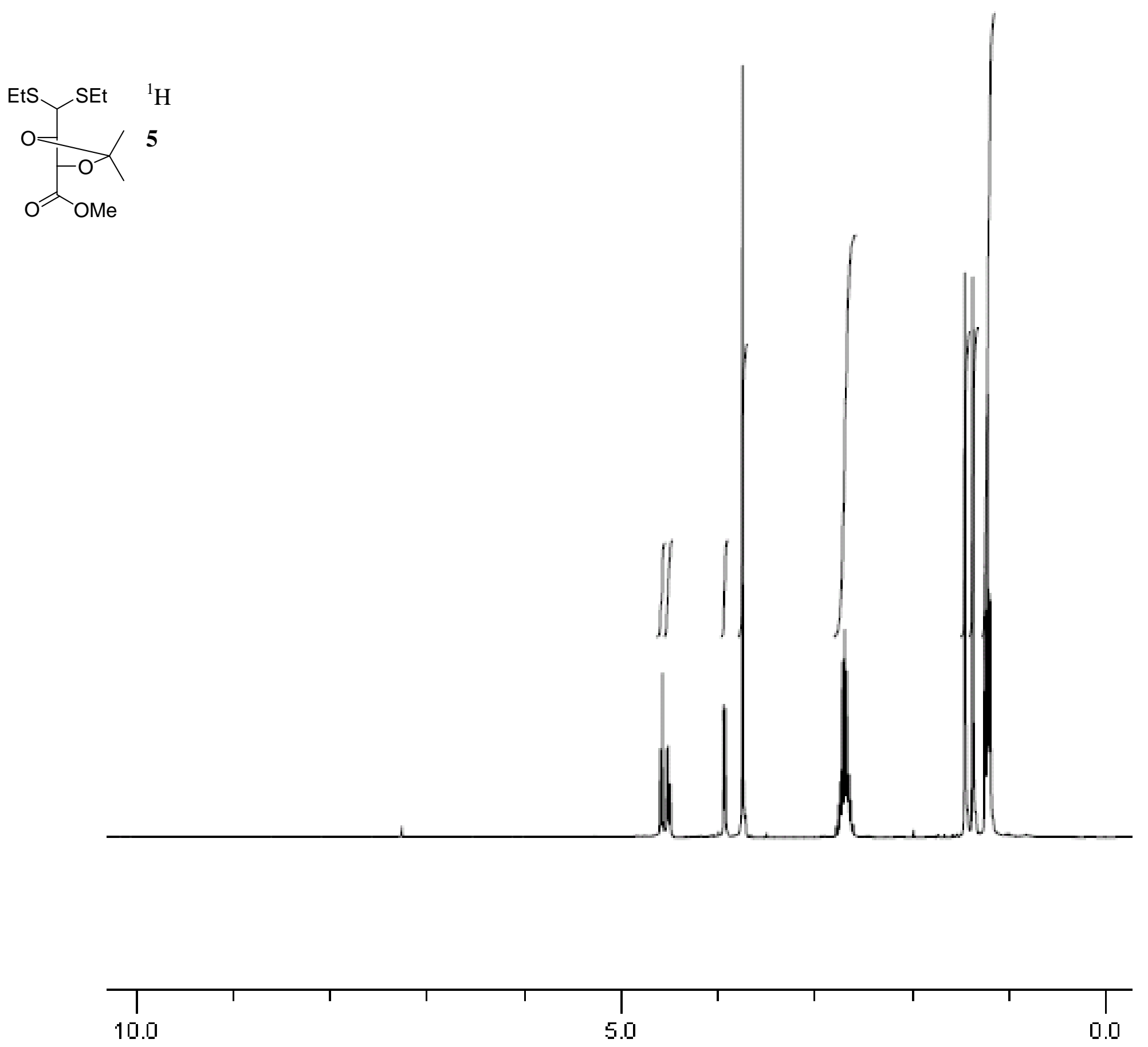

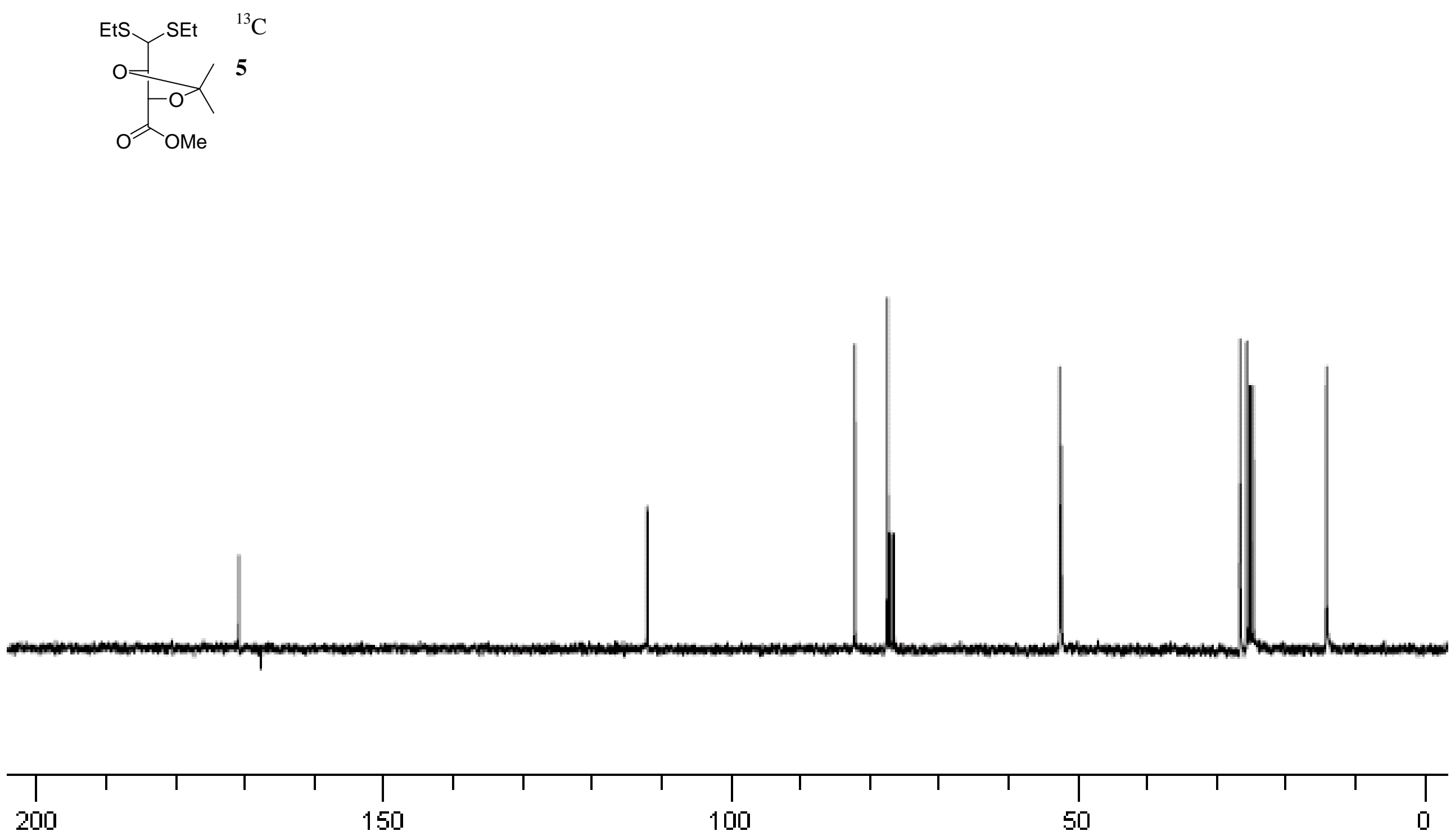

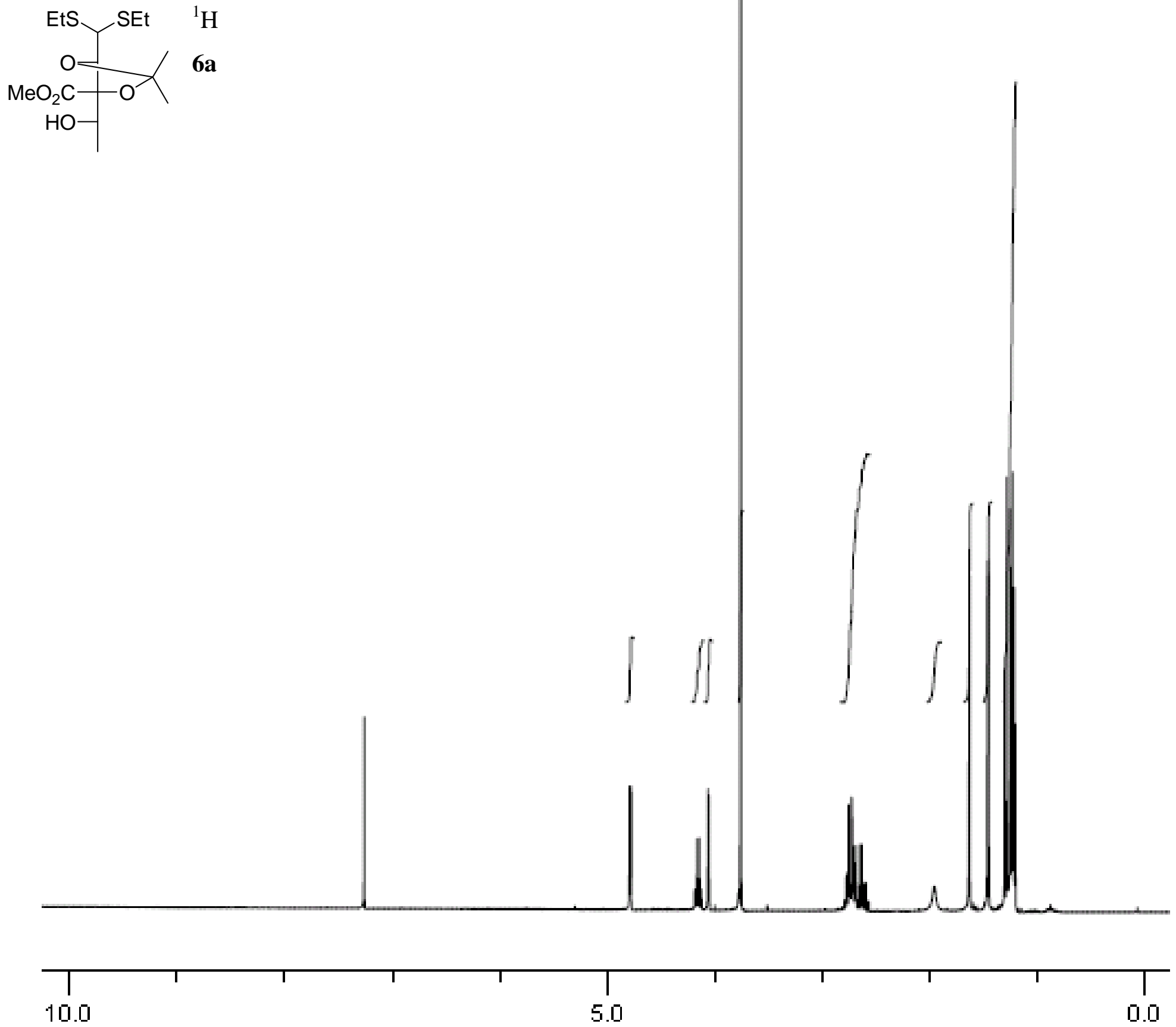

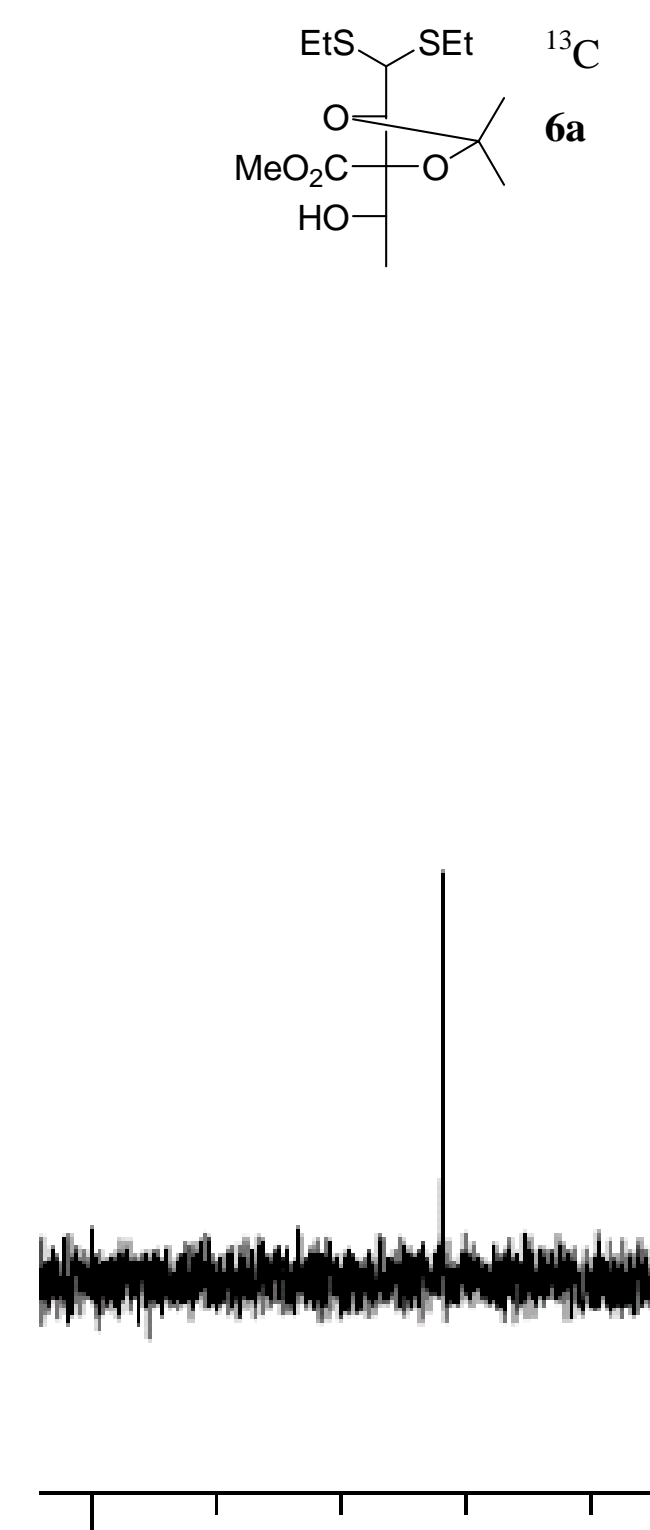

T
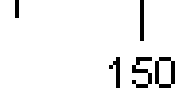

T
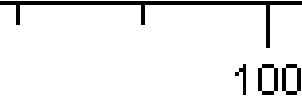

100

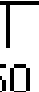

50 

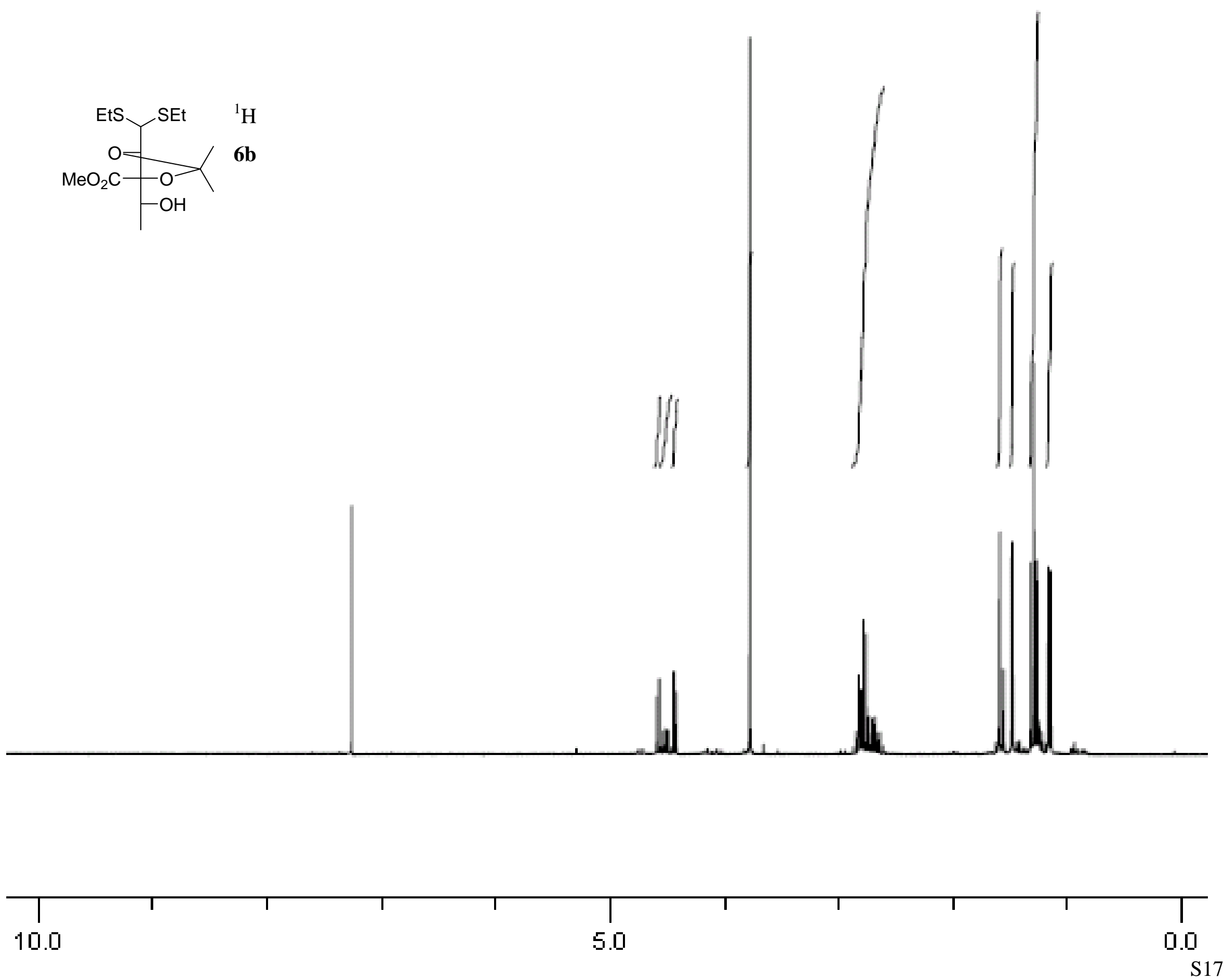

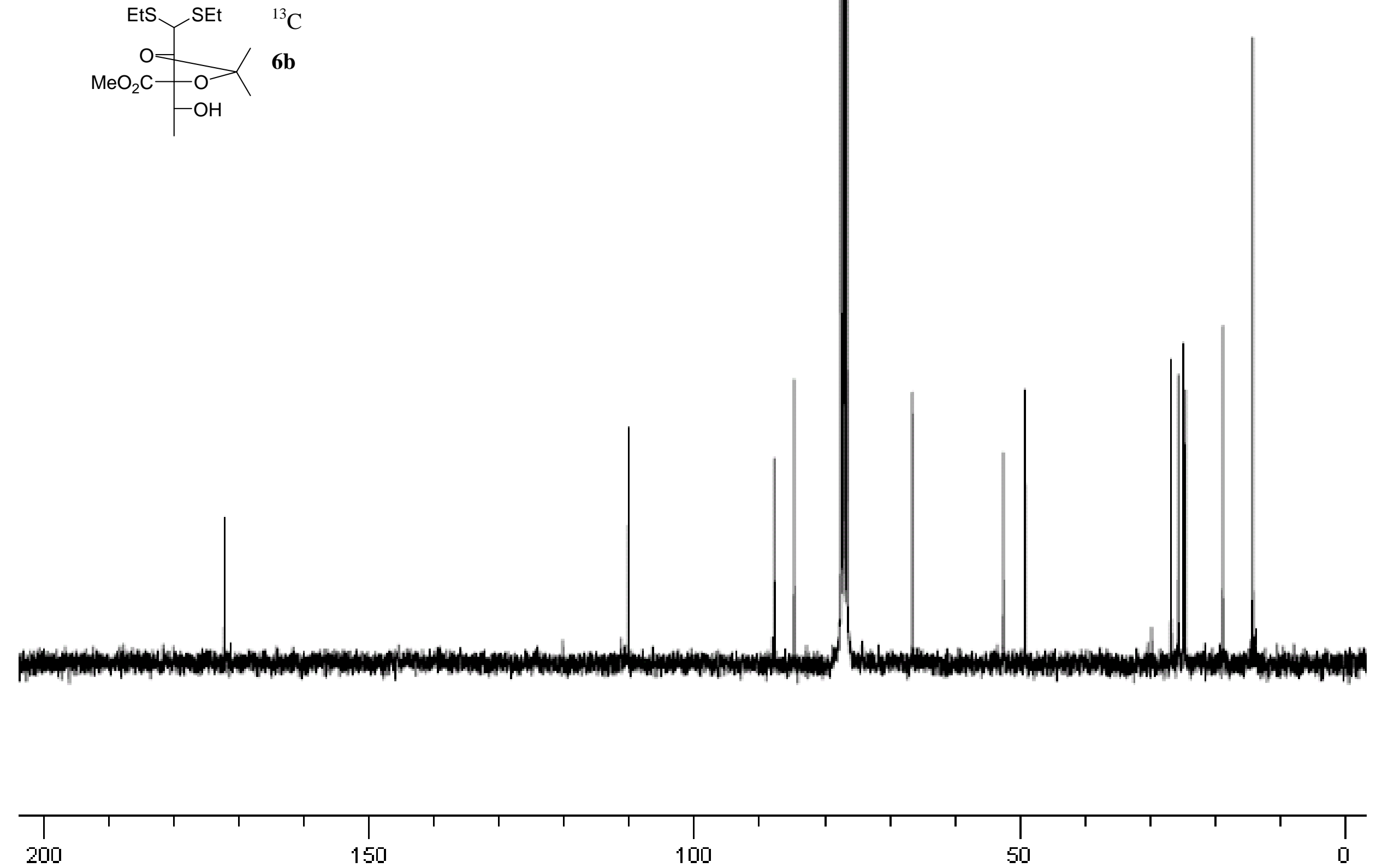
$\underbrace{}_{\mathrm{OHe}}{ }^{7 a}$
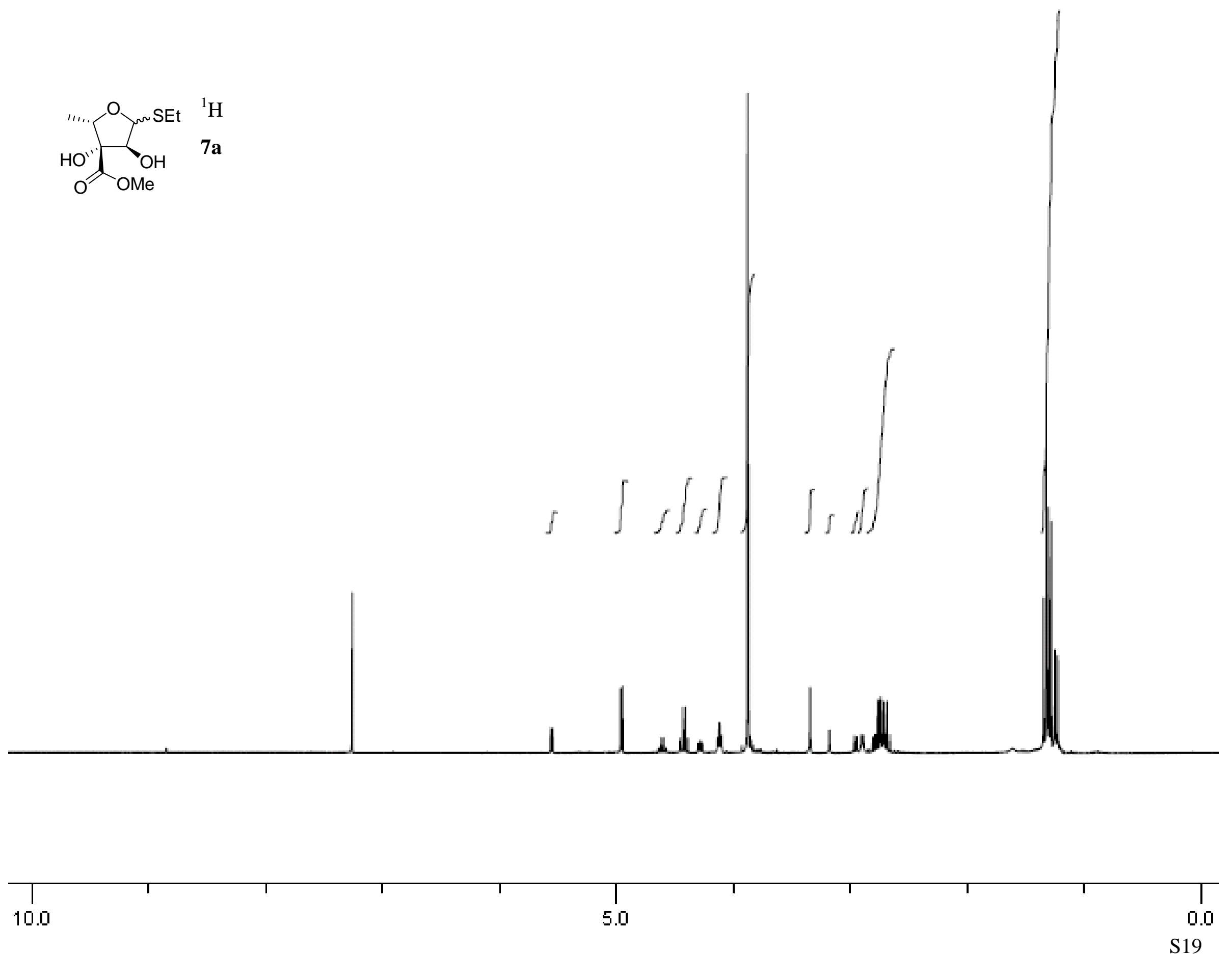

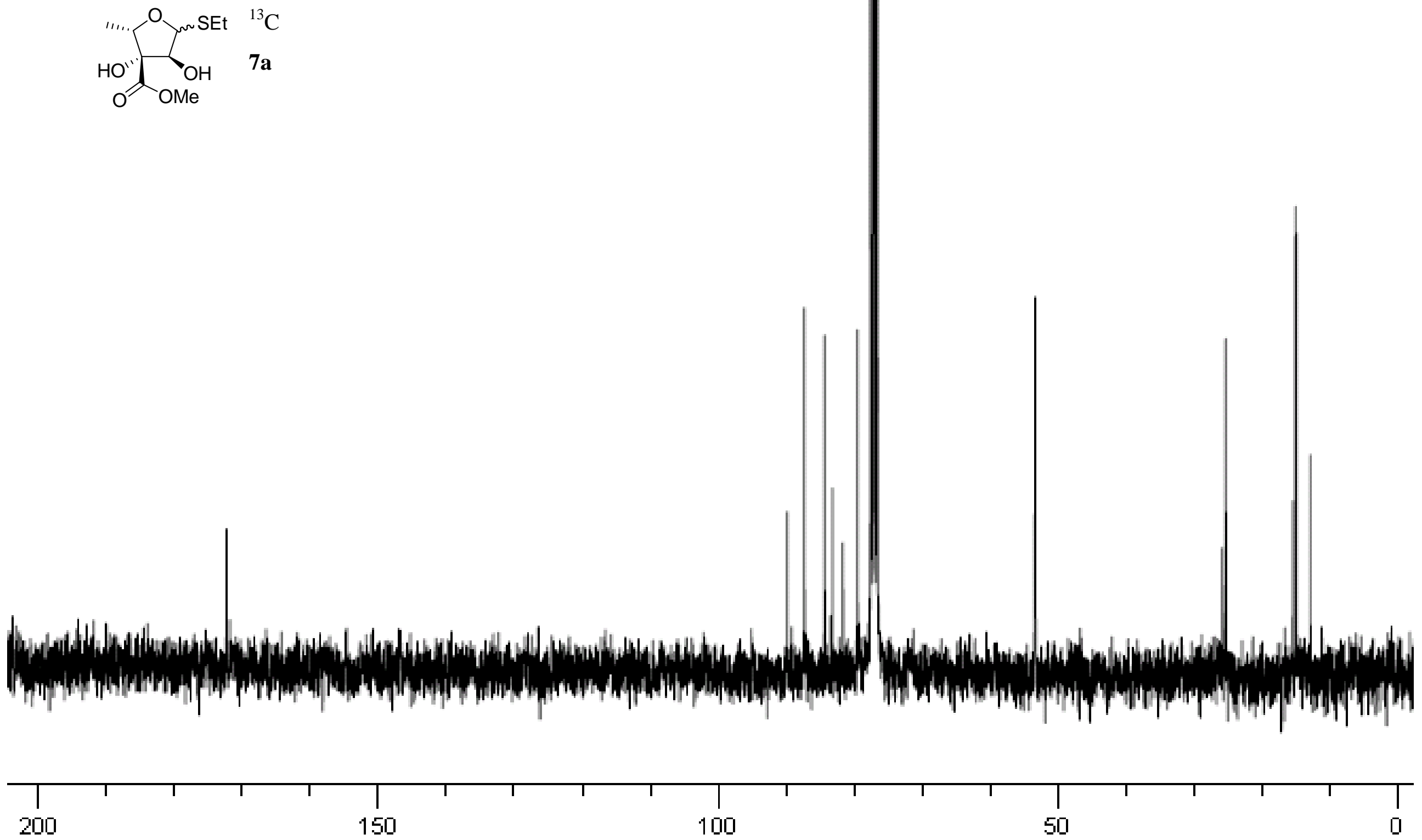
$\underbrace{{ }_{\mathrm{OH}}{ }^{7 b}}_{\mathrm{OMe}}$
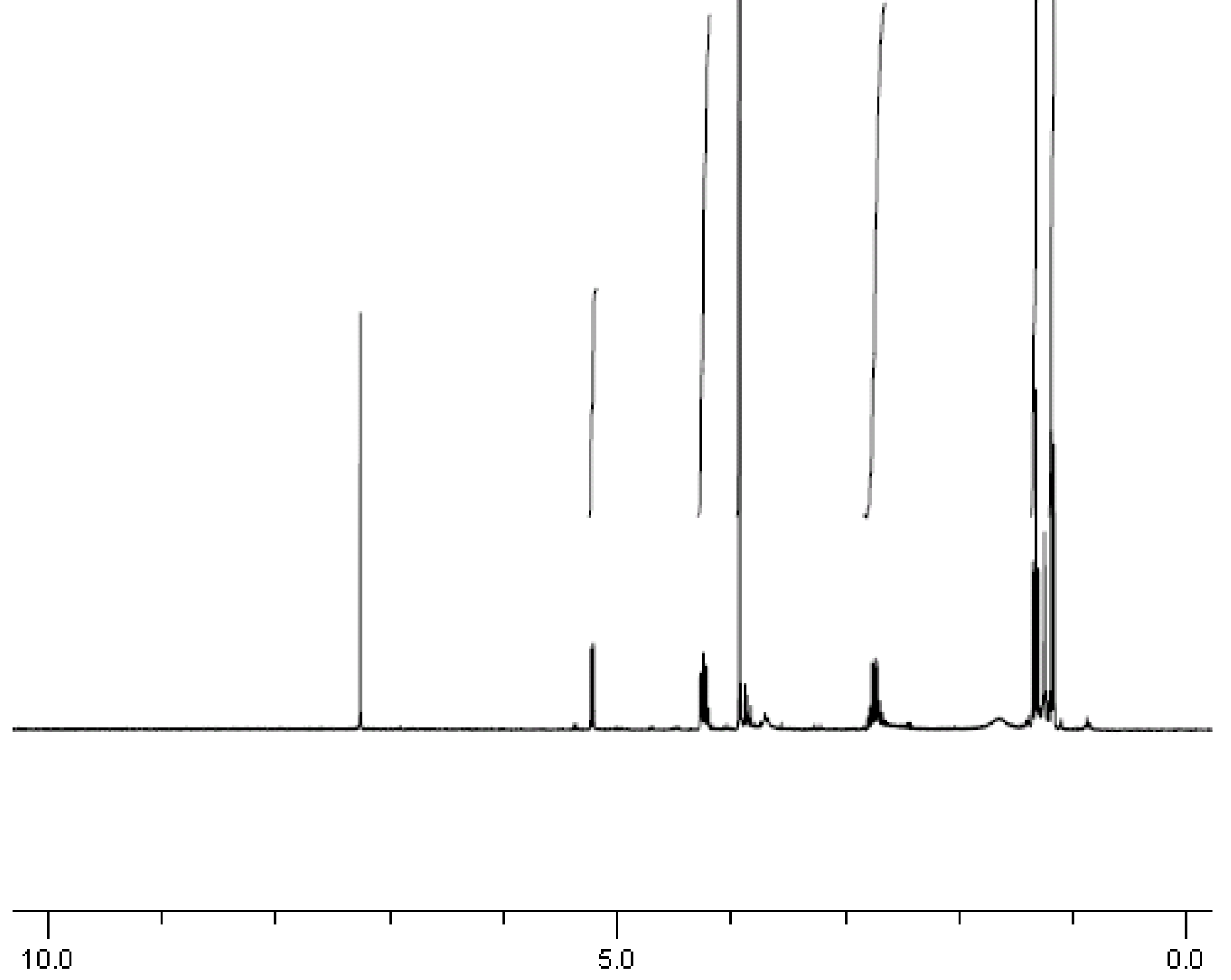


$$
{ }_{\mathrm{OMe}}^{{ }_{\mathrm{OH}}{ }^{7 b}}
$$
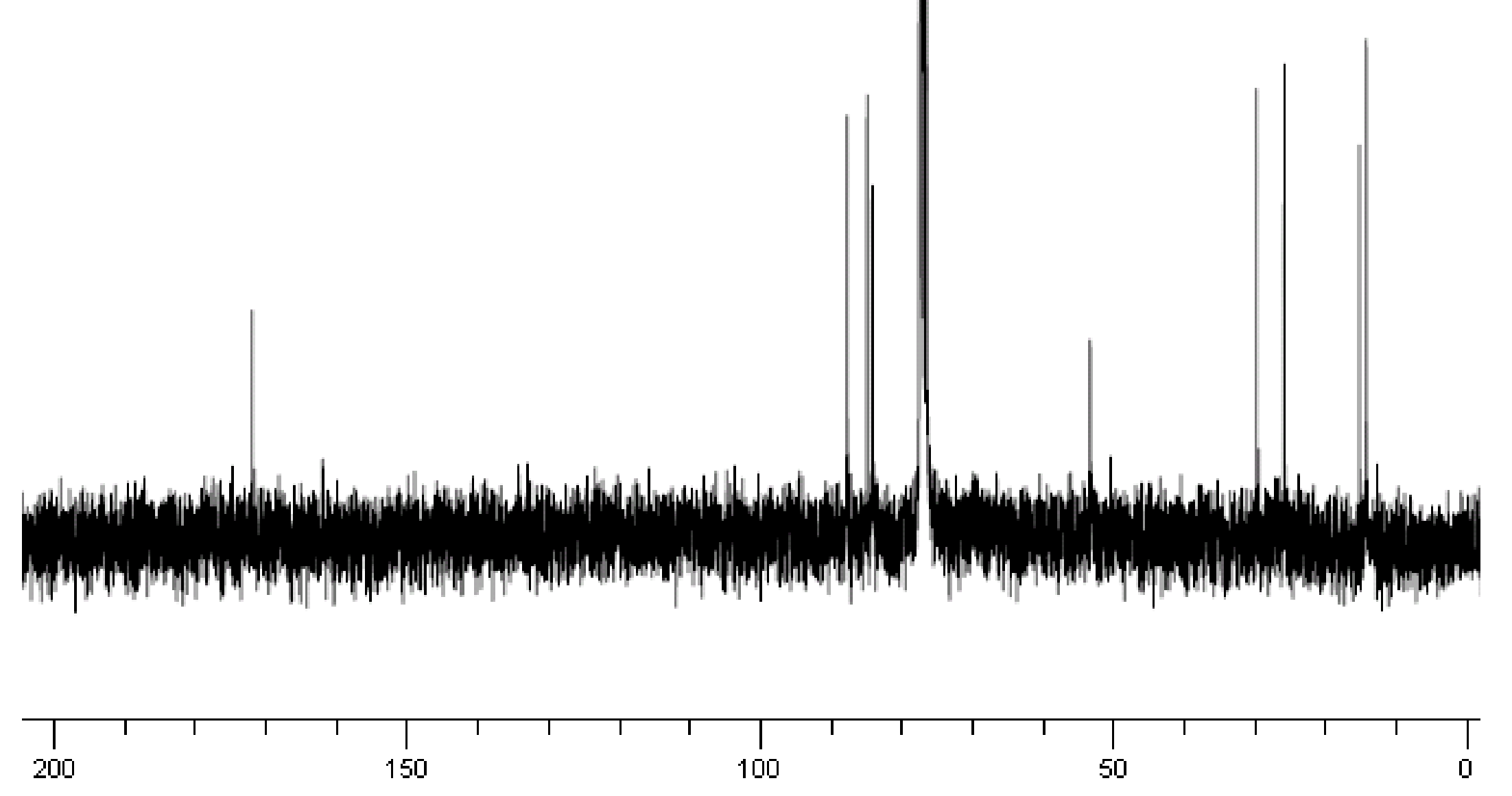

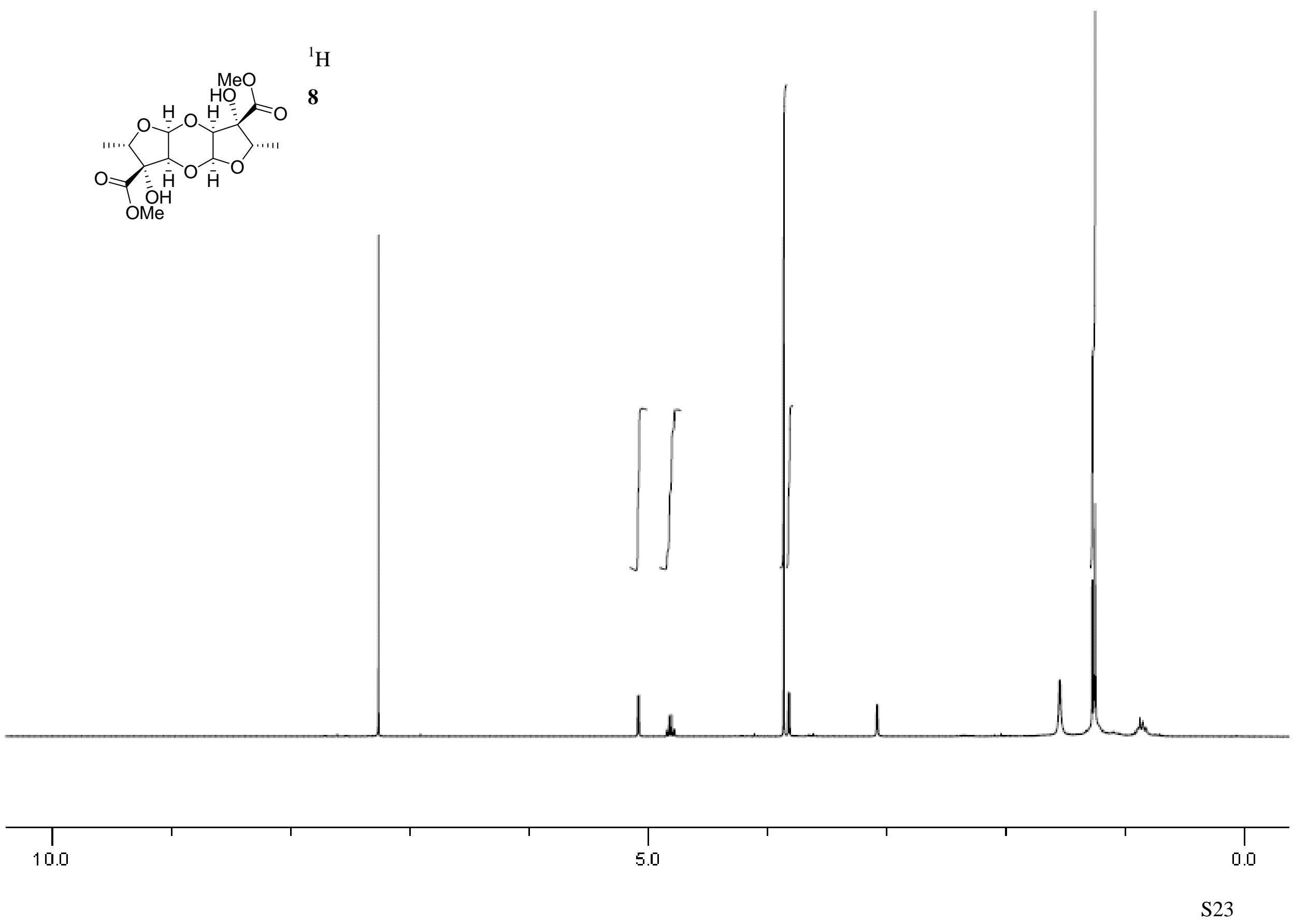

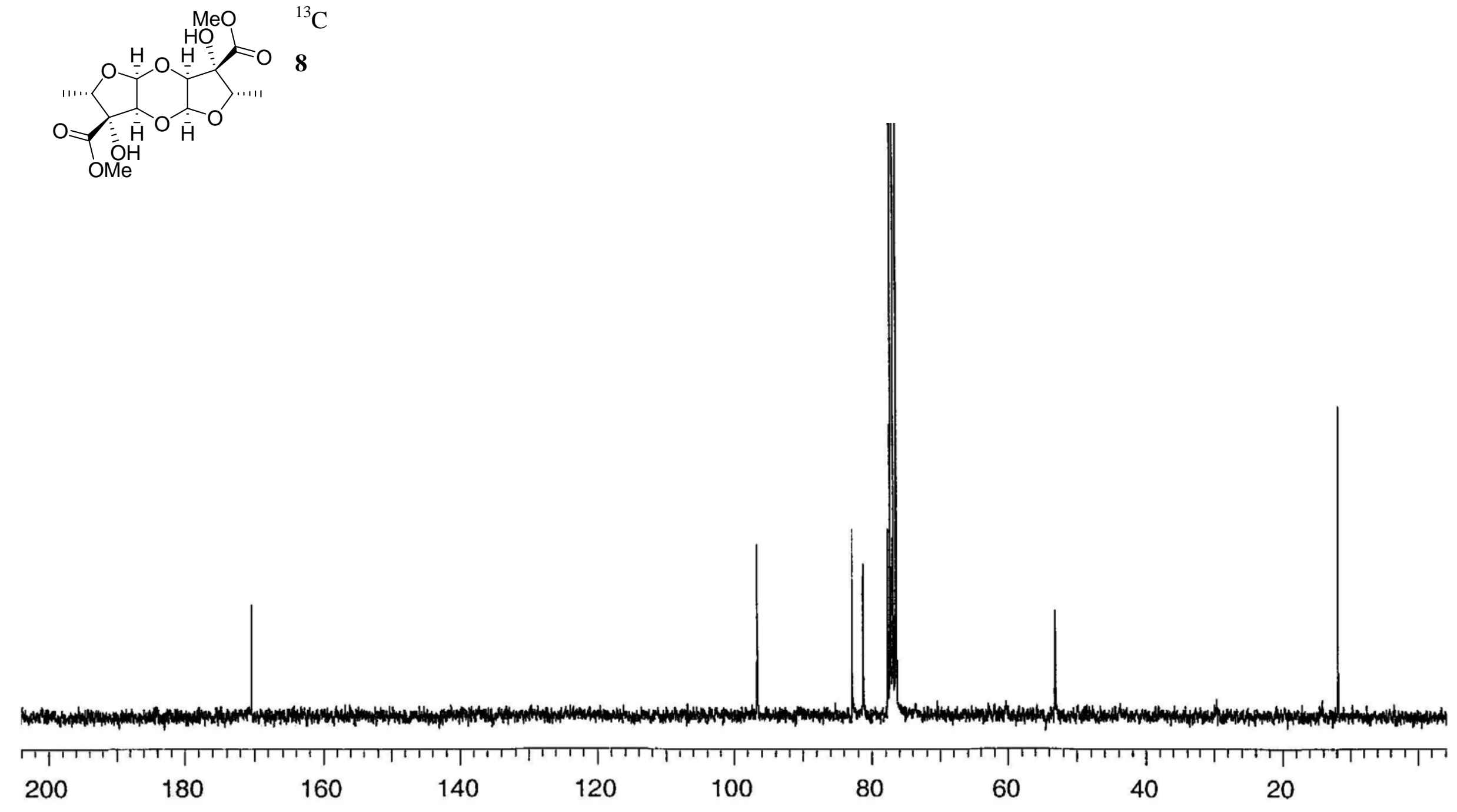


$$
\underbrace{\mathrm{OH}}_{\mathrm{OMe}}{ }^{1} \mathrm{H}
$$
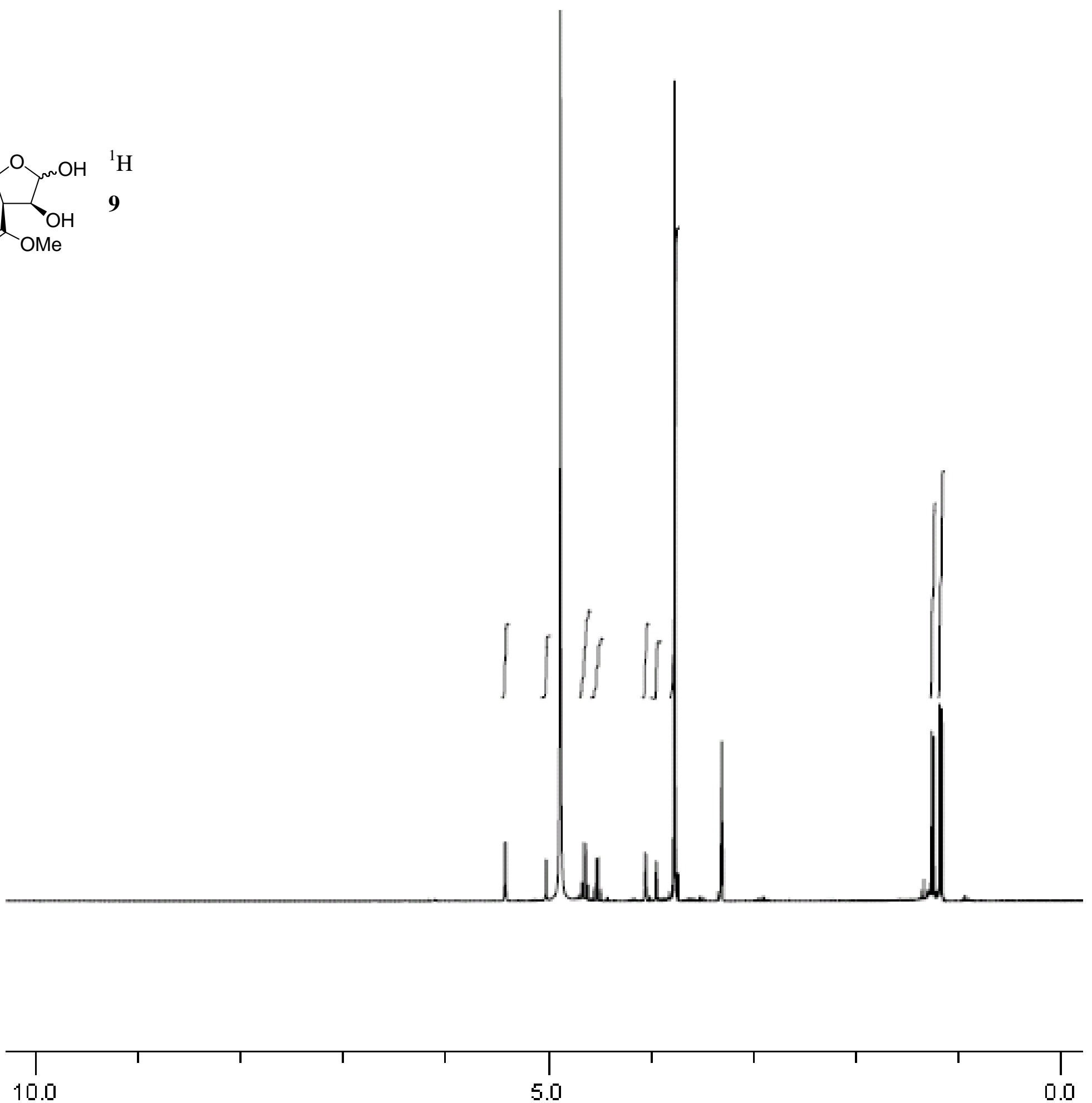

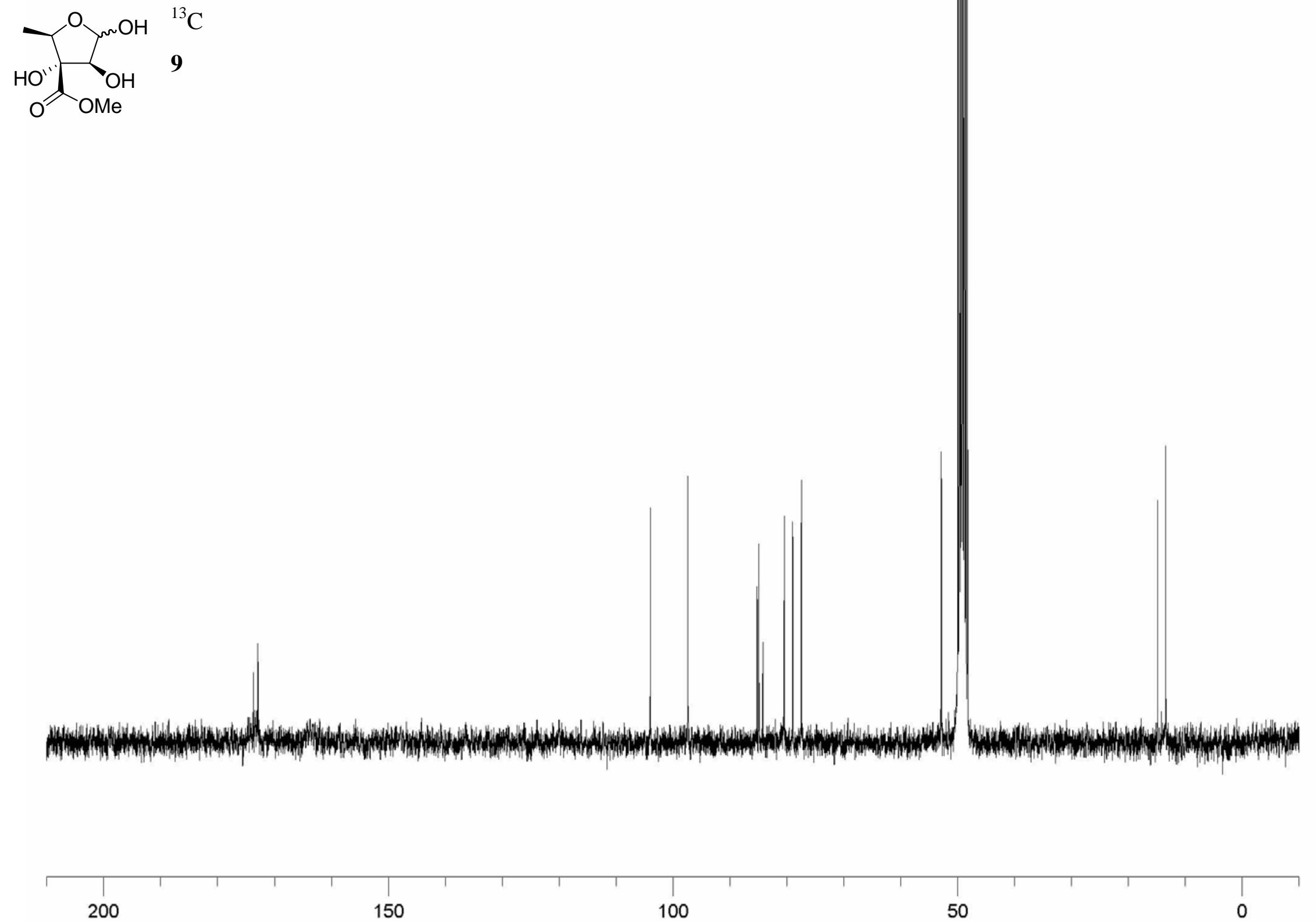
${ }_{\mathrm{OHe}}^{\mathrm{OH}}{ }^{1} \mathrm{H}$
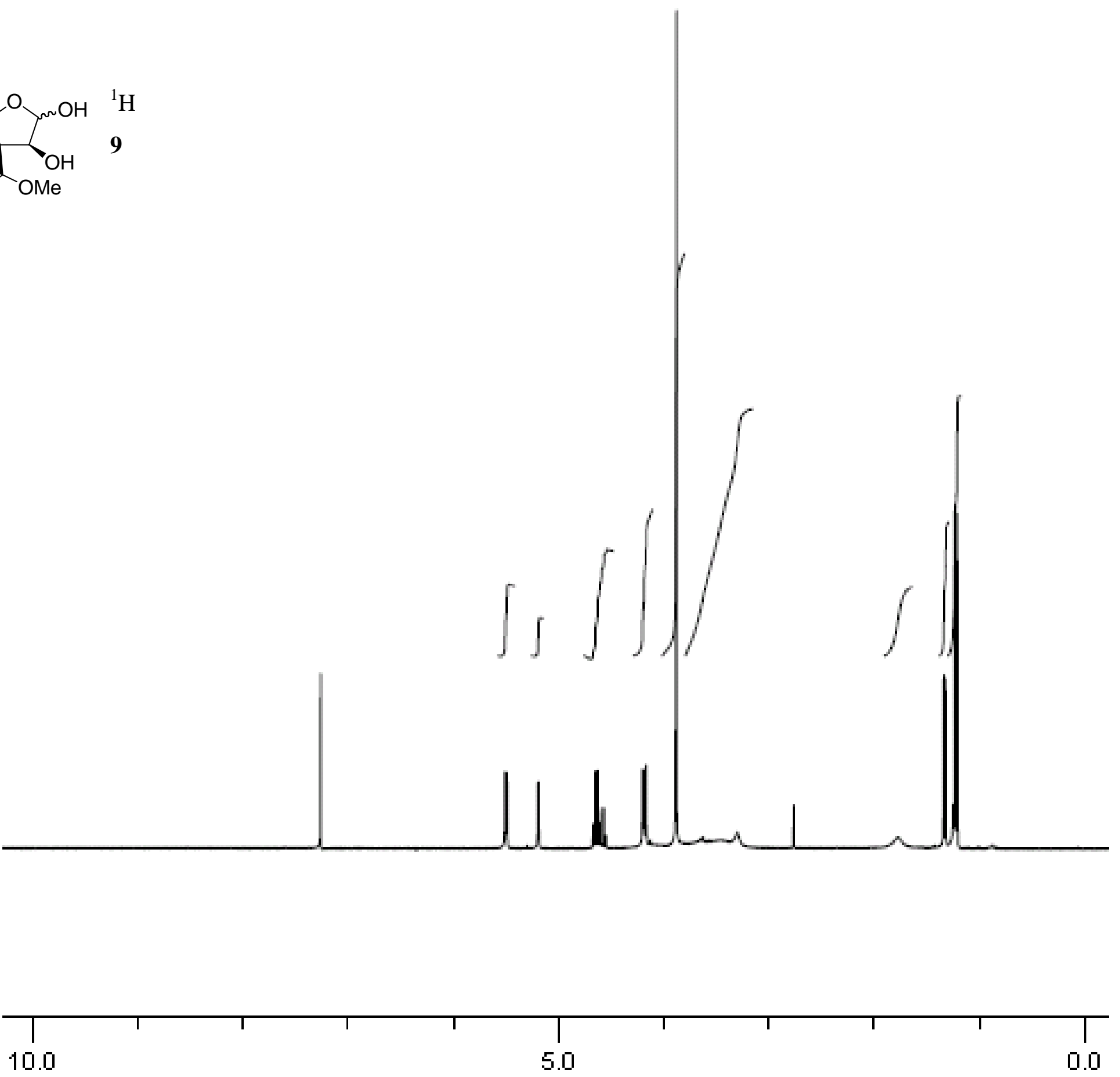


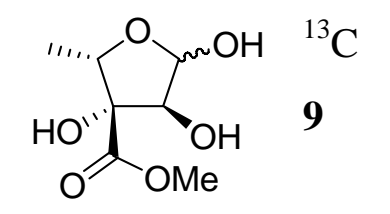

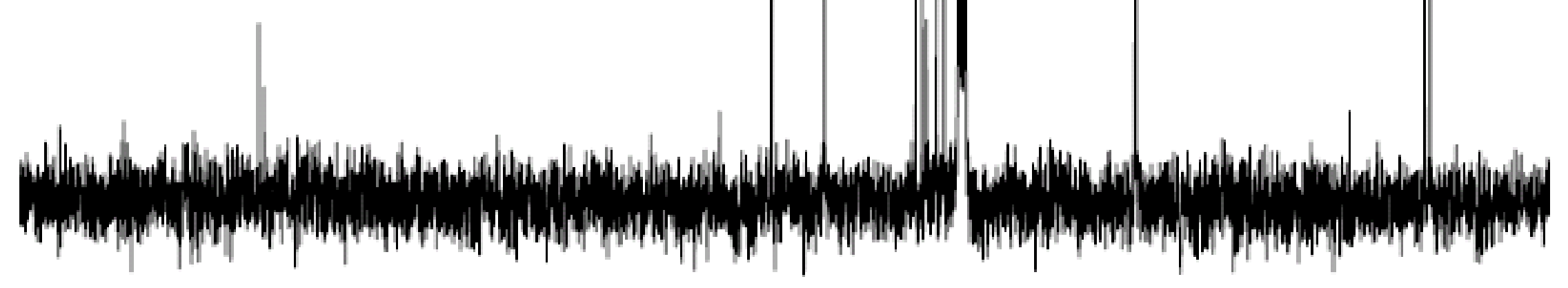

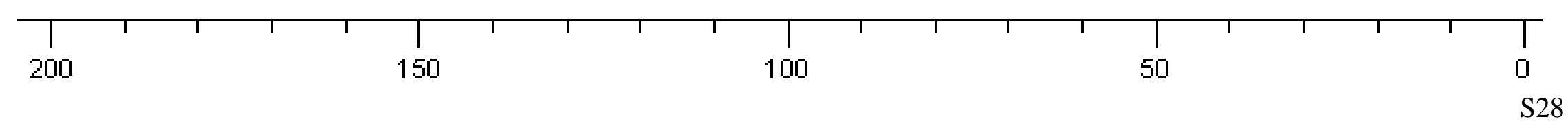




$$
\sum_{\mathrm{OH}}^{\mathrm{OH}}{ }^{1} \mathrm{H}
$$
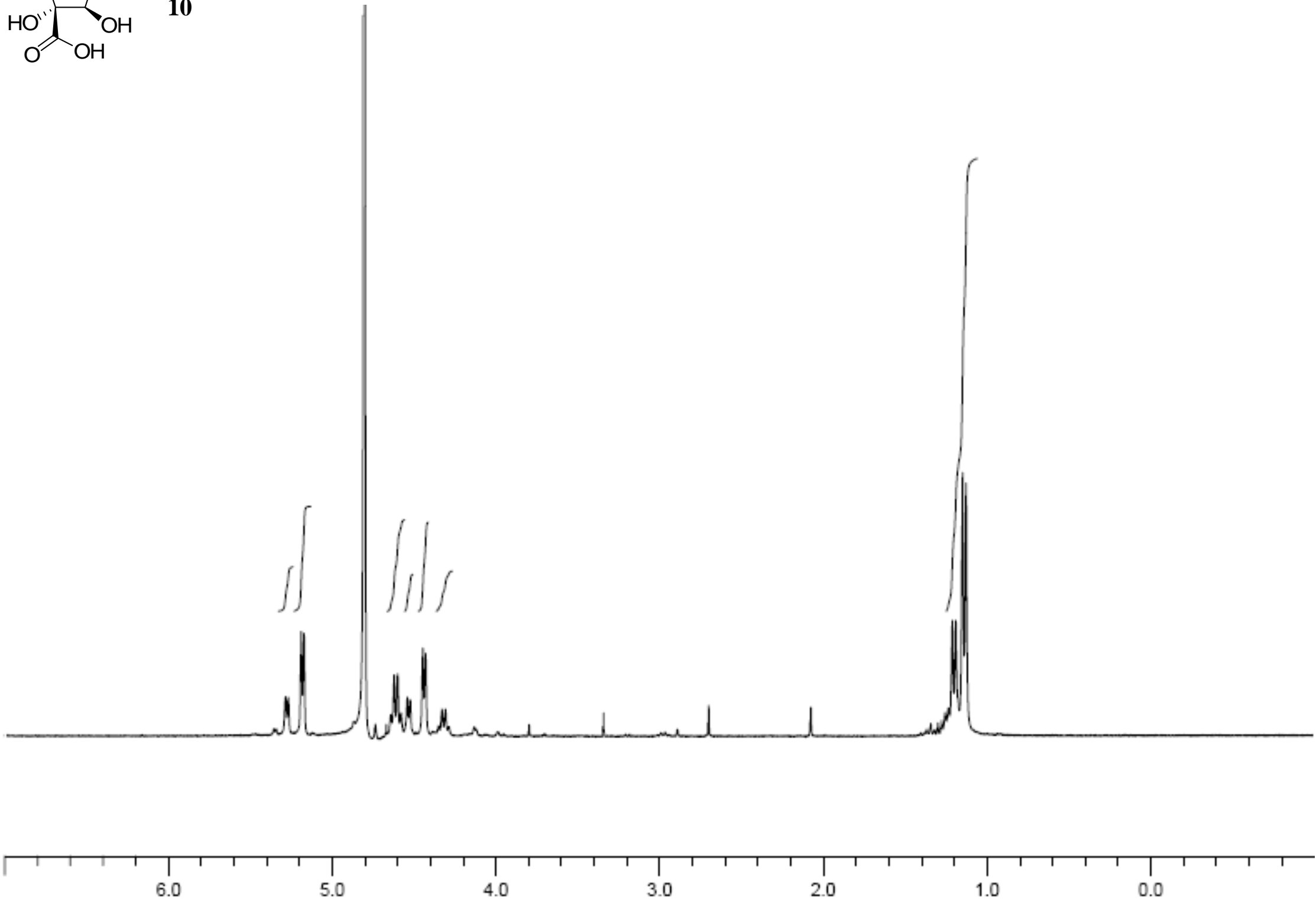


$$
\underbrace{\mathrm{OH}}_{\mathrm{OH}}{ }^{10}
$$

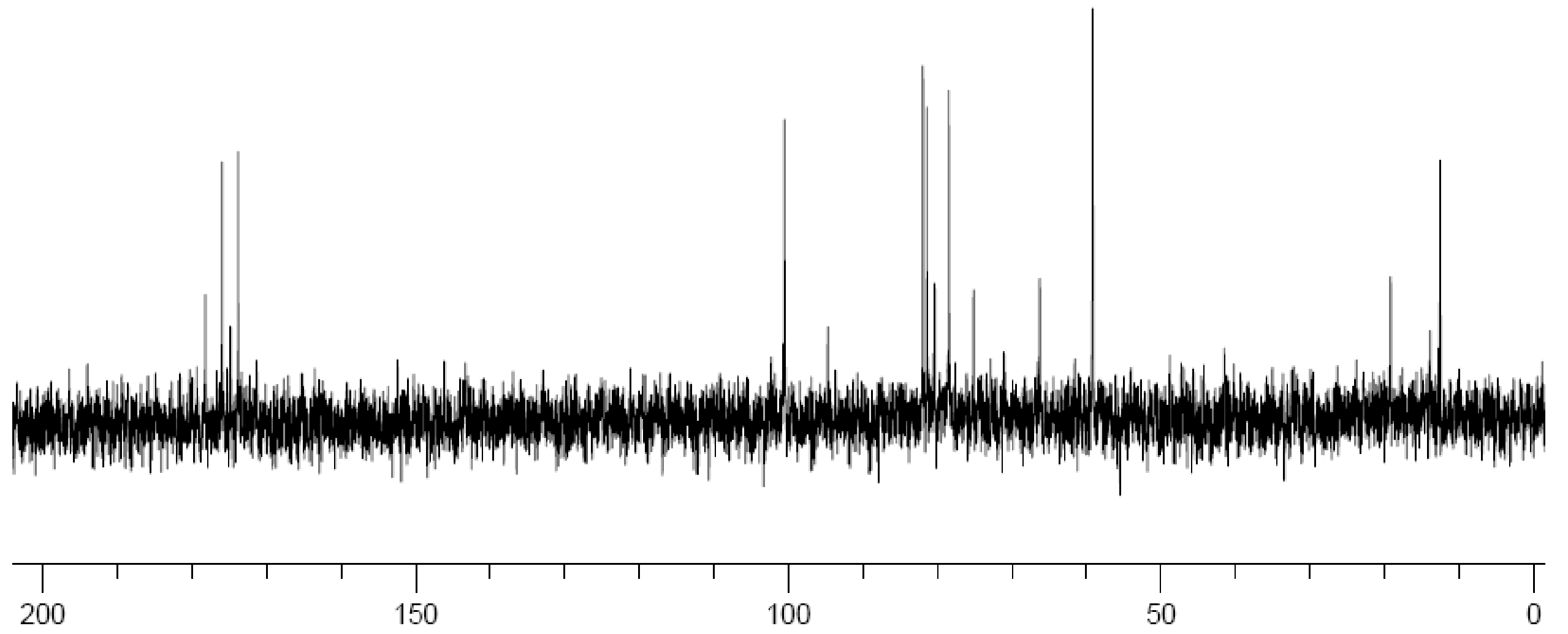


$\sum_{\mathrm{OH}}^{\mathrm{NOH}}{ }^{1} \mathrm{H}$

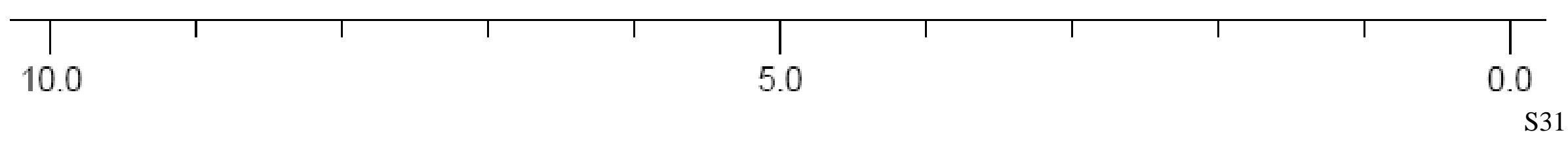



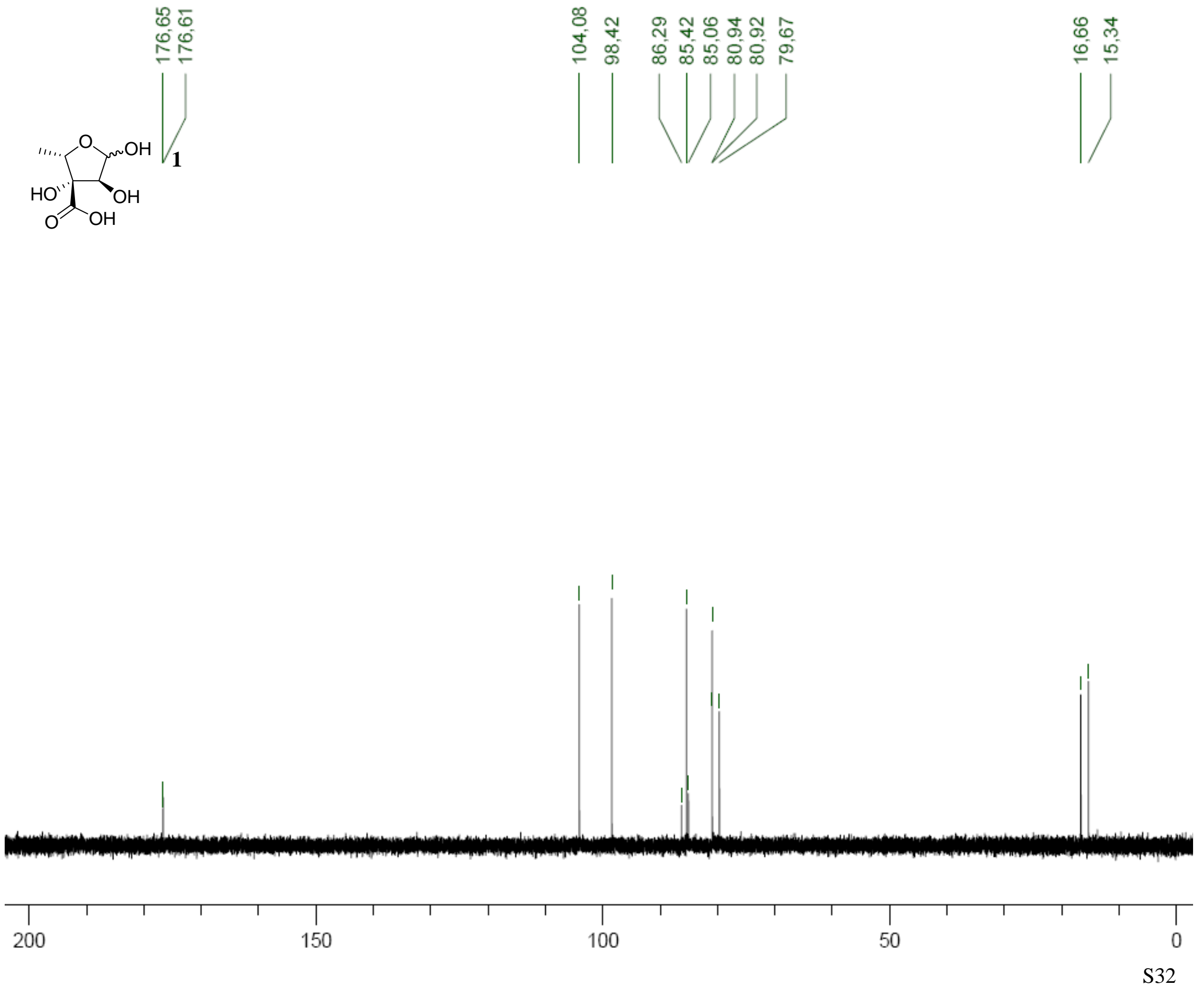


$$
\begin{aligned}
& \text { EtS } \\
& \mathrm{HO}- \\
& \mathrm{MeO}_{2} \mathrm{C}-\mathrm{OH} \\
& \mathrm{HO}
\end{aligned}
$$
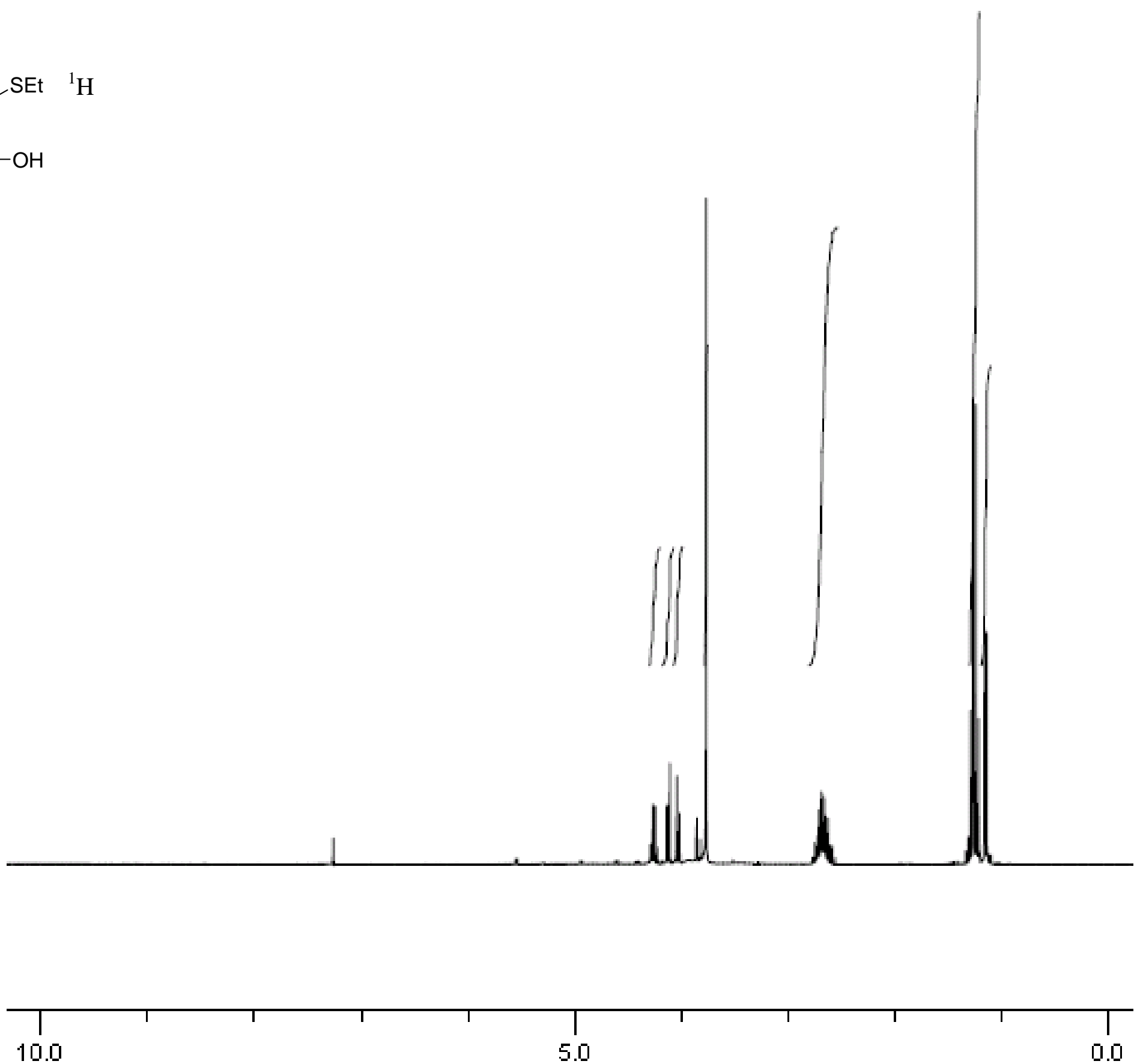

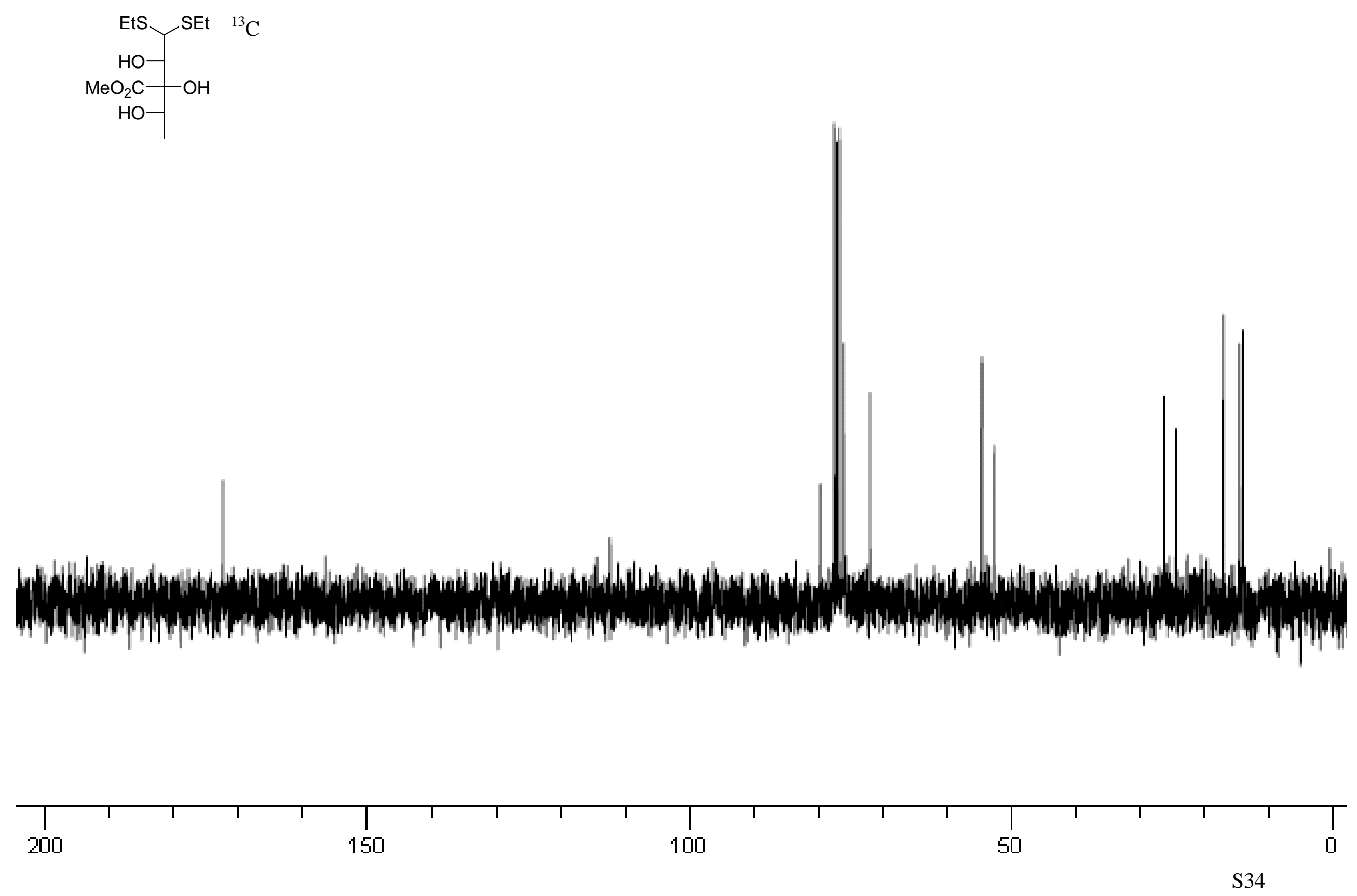


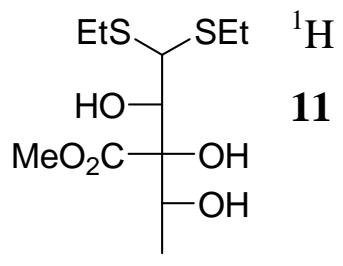
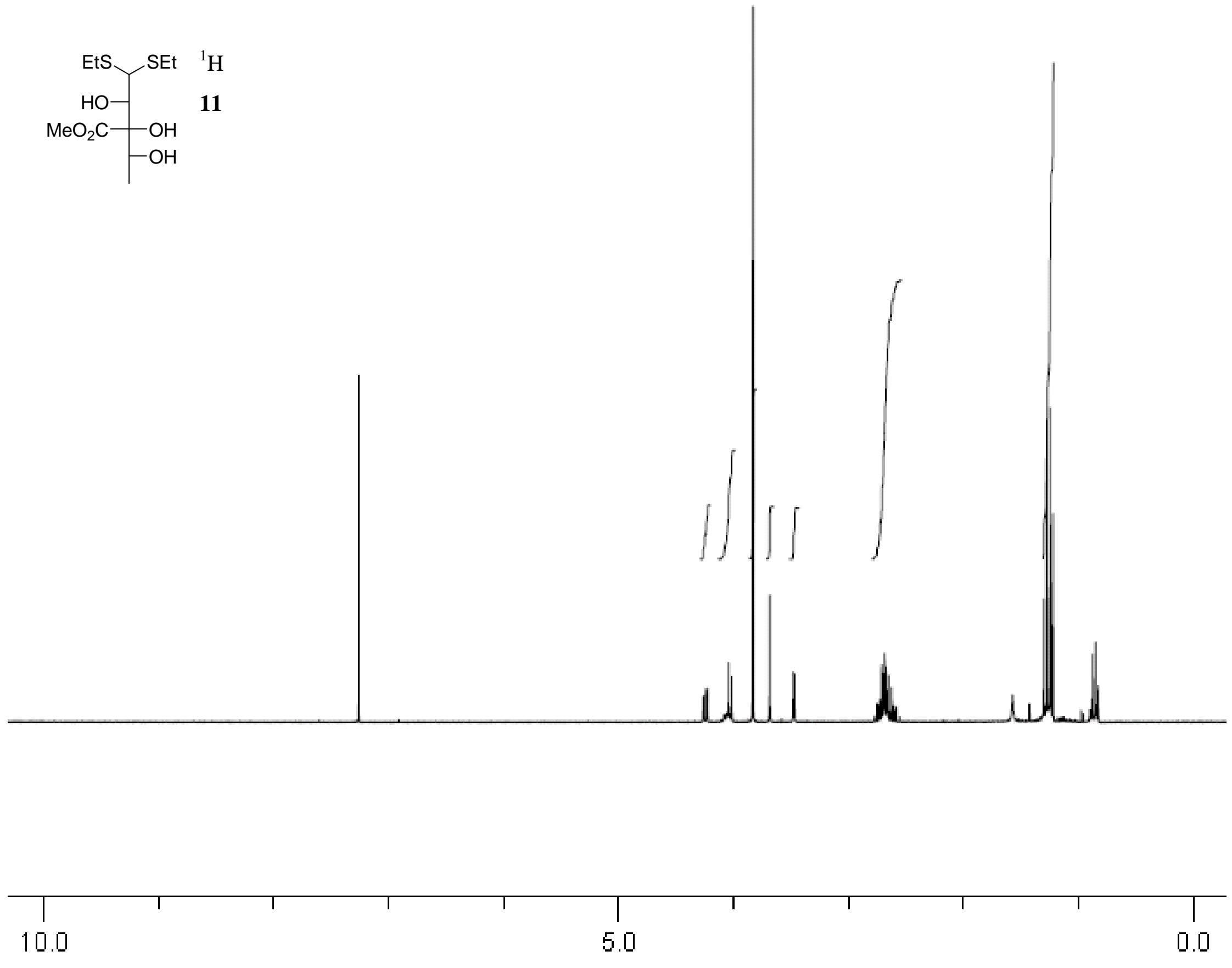


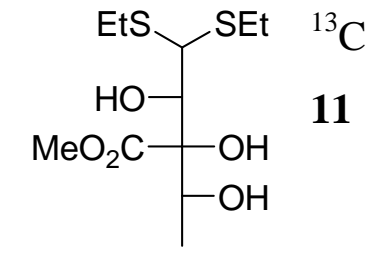

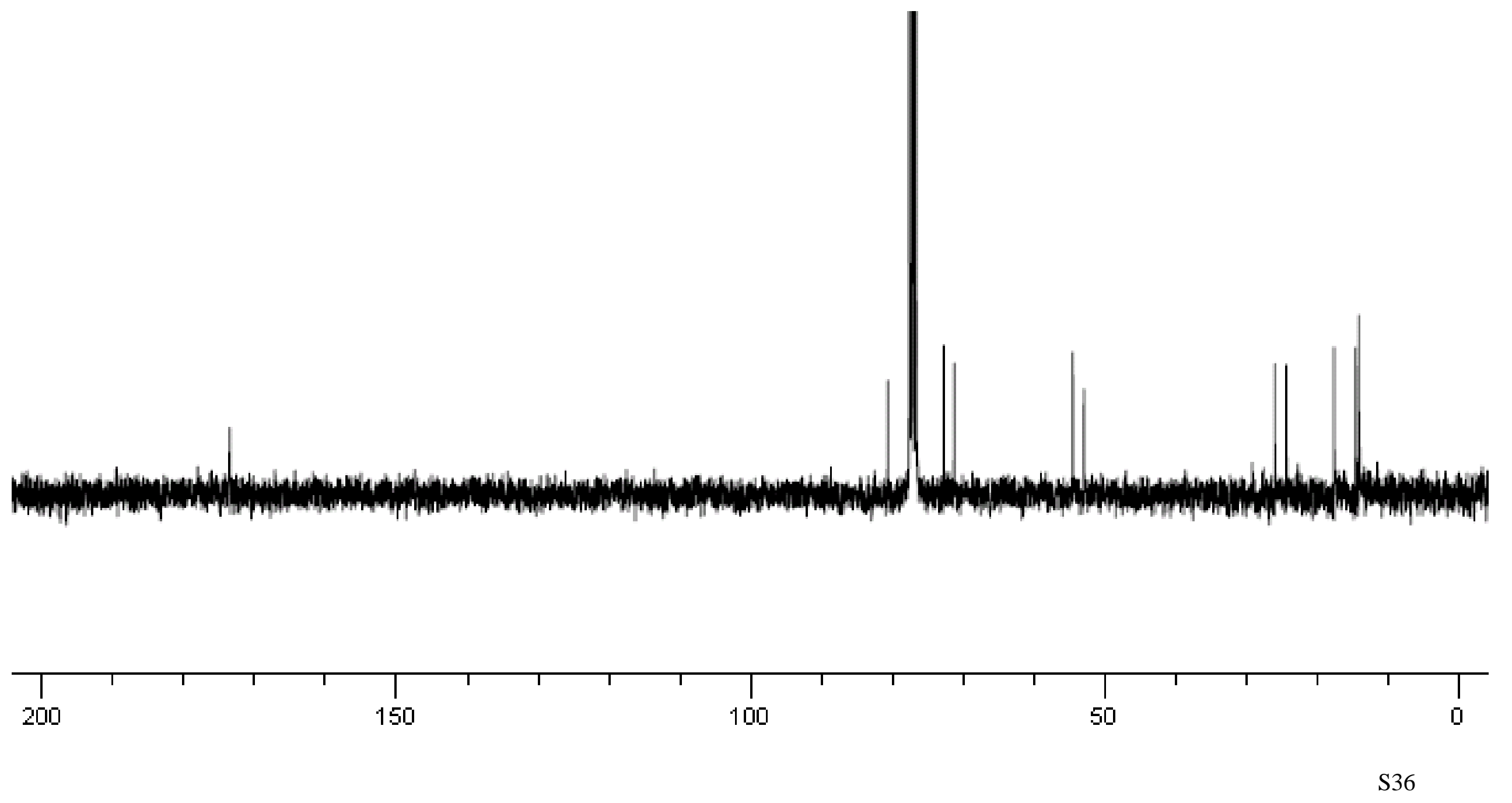


Crystal Structures:

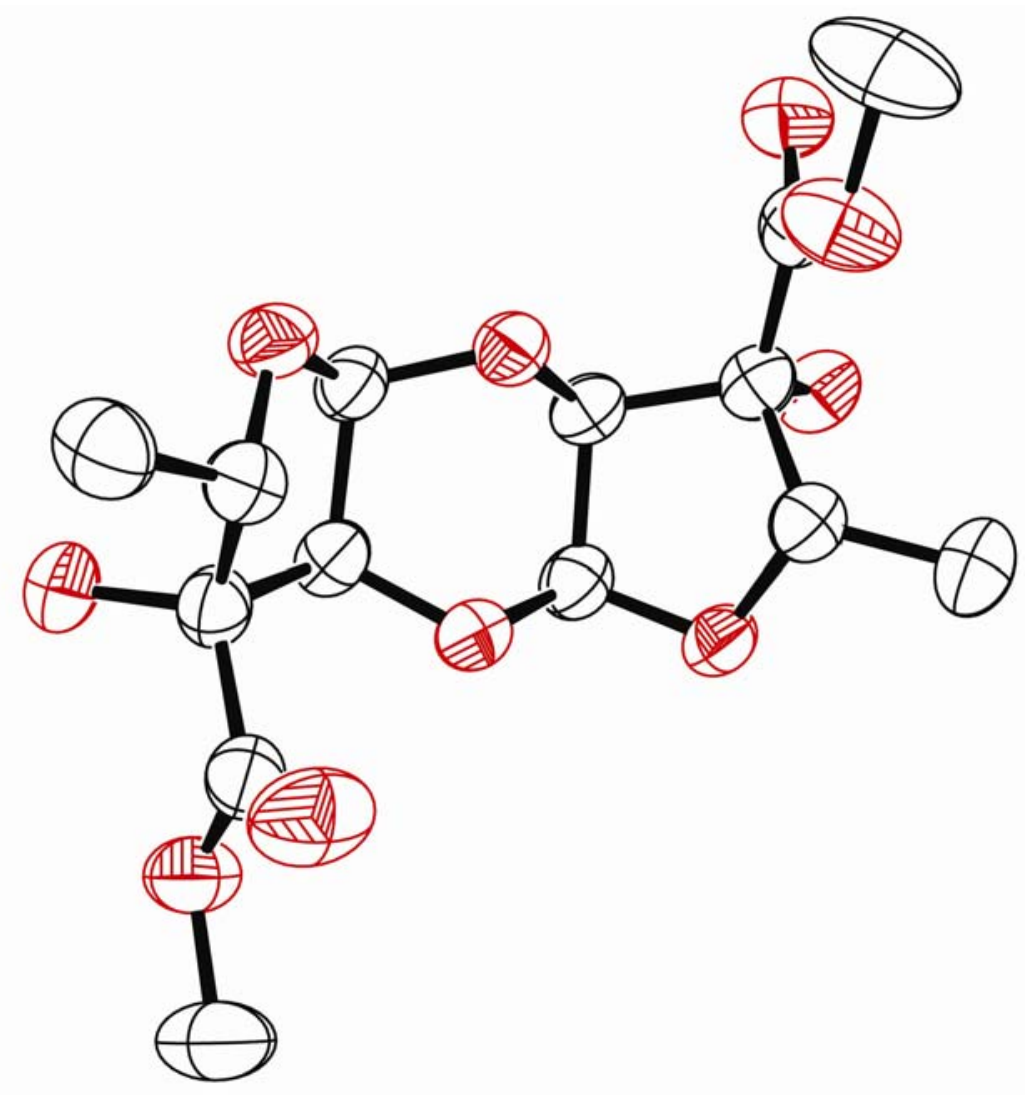

Compund 8

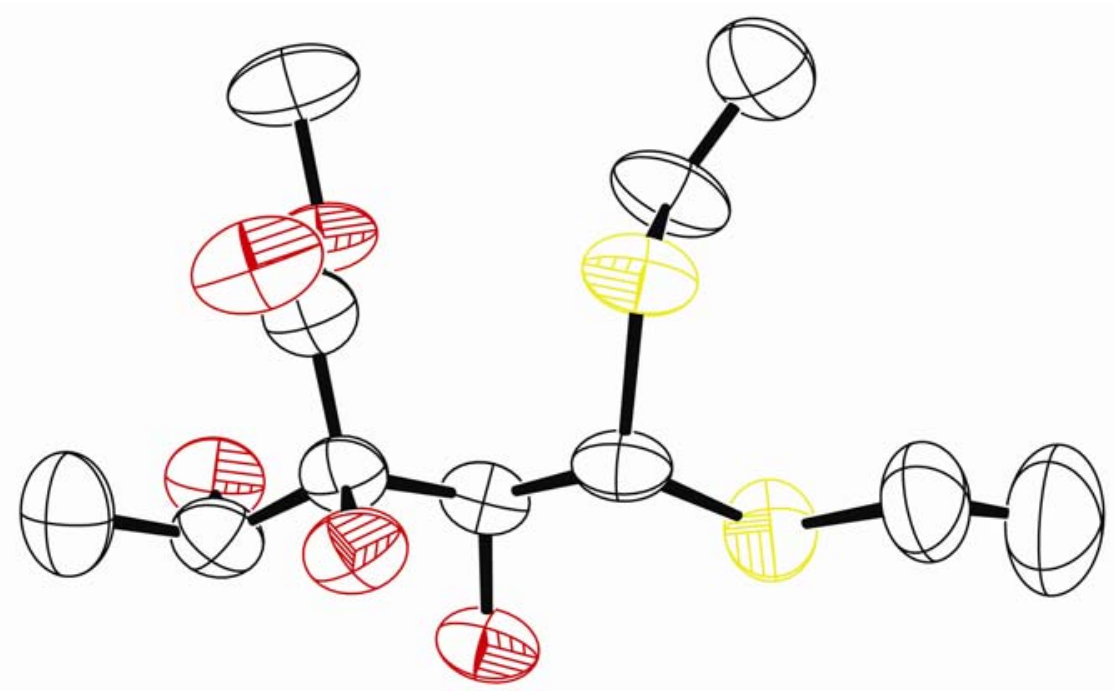

Compund 11 